\title{
1 Global Quantitative Mapping of Enhancers in Rice Genome by
}

\section{STARR-seq}

3

4 Jialei Sun ${ }^{1, \#, \mathrm{a}}, \mathrm{Na} \mathrm{He}^{1, \#, \mathrm{~b}}$, Longjian Niu ${ }^{1,4, \#, *, \mathrm{c}}$, Yingzhang Huang ${ }^{1, \#, \mathrm{~d}}$, Wei Shen ${ }^{2,3, \#, \mathrm{e}}$, $5 \quad$ Yuedong Zhang ${ }^{1, \mathrm{f}}, \mathrm{Li} \mathrm{Li}^{2,3, \mathrm{~g}}$, Chunhui Hou ${ }^{1,{ }^{*}, \mathrm{~h}}$

6

$7 \quad{ }^{1}$ Department of Biology, Southern University of Science and Technology, Shenzhen 8 518055, China

$9 \quad{ }^{2}$ Department of Bioinformatics, Huazhong Agriculture University, Wuhan 430070, 10 China

$11{ }^{3} H u b e i$ Key Laboratory of Agricultural Bioinformatics, Huazhong Agriculture 12 University, Wuhan 430070, China

${ }^{4}$ Department of Biology, Nankai University, Tianjin 300071, China

$14 \quad$ Equal contribution.

*Corresponding authors.

Email: niulj@mail.sustc.edu.cn (Niu L), houch@sustc.edu.cn (Hou C).

Running title: Sun J et al / Global rice enhancer identification

a ORCID: 0000-0001-9320-3701.sunj1@mail.sustc.edu.cn

b ORCID: 0000-0002-6179-9552. hena@ sustc.edu.cn

c ORCID: 0000-0002-2545-3669. niulongjian@ 126.com

d ORCID: 0000-0003-1876-1089. huangyz@mail.sustc.edu.cn

e ORCID: 0000-0002-3585-0369. shen_bioinformatics@webmail.hzau.edu.cn

f ORCID: 0000-0002-6179-9552. zhangyd@ mail.sustc.edu.cn

g ORCID: 0000-0002-9594-8341. 1i.li@mail.hzau.edu.cn

h ORCID: 0000-0002-8339-1857. houch@ sustc.edu.cn

(Total 8,276 words, 7 figures, 10 supplementary figures, 3 supplementary tables and 5 supplementary lists.) 
30

31

32

\section{1} (2)

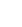
55 56 57 8

\section{Abstract}

Identification of enhancers has been a challenge in plants. STARR-seq measures enhancer activity of millions fragments in parallel. Here we present a global map of rice enhancers quantitatively determined using STARR-seq. Most enhancers are mapped within genes, especially at the 5' untranslated regions (5' UTR) and the coding sequences. Silent and low expressed genes in genomic regions enriched with transposable elements (TEs) are frequently found containing proximal enhancers. Analysis of enhancer epigenetic features at their endogenous loci revealed that most enhancers do not co-localize with DNase I hypersensitive sites (DHSs) and are lack of H3K4me1. Clustering enhancers by their epigenetic modifications revealed that about $40 \%$ of identified enhancers carry one or more epigenetic marks. Repressive $\mathrm{H} 3 \mathrm{~K} 27 \mathrm{me} 3$ is frequently enriched with positive marks of $\mathrm{H} 3 \mathrm{~K} 4 \mathrm{~m} 3$ and/or H3K27ac, which together may bookmark poised enhancers. Intergenic enhancers were predicted based on the location of DHS relative to genes, which overlap poorly with functionally identified enhancers. In summary, enhancers were quantitatively identified by functional analysis in a model plant genome, which provides a valuable resource for further mechanistic studies in different biological contexts.

KEYWORDS: Rice; Enhancer; STARR-seq; Quantification; Functional analysis 


\section{Introduction}

Gene expression is tightly regulated, which is critical for plant development and responses to changes in environment and hormones [1]. Promoters at transcription start site (TSS) are frequently considered sufficient for the initiation and elongation of transcription, but the level of promoter-driven expression is generally low [1]. High level of gene expression requires the participation of enhancers to increase the efficiency of transcriptional initiation and elongation to produce more mRNAs, though the exact mechanisms are still poorly understood.

Enhancers are generally believed to regulate their target genes through the recruitment of transcription factors (TFs) and function in a distance- and orientation-independent manner [2]. In mammalian genomes, one gene can be regulated simultaneously by multiple enhancers or by different enhancers in different tissues or at different developmental stages. Moreover, one enhancer can also regulate multiple genes [2-5]. The lack of specific location relationship with target genes makes the identification of enhancers more difficult than promoters.

The advancements in molecular biology and computational techniques have enabled the characterization of enhancers genome-widely based on epigenetic marks [6-15] or direct measurement of transcription enhancing activity of candidate sequences [16-22]. Intergenic enhancers have been predicted in Arabidopsis according to the location of DNase I hypersensitive site relative to proximal genes [23]. However, the arbitrary exclusion of DNase I sites within $1.5 \mathrm{~kb}$ upstream of TSS and gene body may exclude substantial amount of potential enhancers. So far, only a handful of enhancers have been identified in plants [24-28], let alone a genome-wide annotation of enhancers based on functional analysis.

Measuring enhancer activity of millions fragments had been successfully 
achieved using STARR-seq in the Drosophila melanogaster and mammalian genomes $[16,18-20,22]$. We used STARR-seq to map potential enhancers globally in a rice genome. The accuracy of large scale quantification analysis of candidate sequences was demonstrated in a plant system. Furthermore, we revealed some epigenetic characteristics which may be unique for identified enhancers in rice genome. Finally, our results provided a regulatory element resource for further functional and mechanistic studies in different biological contexts.

\section{Results}

\section{Global quantitative enhancer discovery using STARR-seq}

To comprehensively identify sequences with enhancer activity, we constructed a reporter library from randomly fragmented genomic DNA of rice cultivar Nipponbare (Oryza sativa L. ssp japonica). The plasmids DNA of reporter library were transfected into protoplasts isolated from the stem of rice seedlings grown for 2 weeks in replicates and plasmids and mRNA were isolated 16 hours later. Sequencing libraries of plasmid and mRNA were generated and sequenced on an Illumina platform (Figure 1A). For two transfections, 15.5 million and 30.6 million (sTable 1) independent fragments were recovered with a median length of $\sim 670$ base pairs from input plasmid libraries (sFigure 1A) by Illumina paired-end sequencing. It covered $\sim 90 \%$ of both repetitive and non-repetitive genome sequences with at least one unique fragment (sFigure 1C and 1E). For the cDNA libraries generated from isolated mRNAs, 6.1 million and 13.7 million of independent fragments were produced (sTable 1). The libraries were quality checked and the enrichment of cDNA over input plasmid was determined for each 600 bin and potential enhancers were identified (sFigure 2-6) and an example was shown in Figure 1B.

This method yielded 15,208 and 12,210 regions (Figure 2A) that were significantly enriched $[\mathrm{p}<0.001]$ from two replicates, respectively. Pearson correlation 
117 coefficient for the two replicates is 0.604 (sFigure 4), which indicates that

118 STARR-seq in plant system is reproducible. Only enriched peaks identified in both 119 biological replicates, totally 9,642 sites (sList 1), were kept for further analysis (Figure 2A). To validate the identified peaks, we tested 22 sites (sList 2) chosen across a wide range of enrichments by cloning them into luciferase reporter vector and quantified the reporter gene expression by reverse transcription and real-time PCR (RT-PCR) and normalized against the expression of co-transfected Renilla reporter gene. STARR-seq enrichment and RT-PCR quantification were strongly related from the entire activity range for enhancers from weak to strong (Pearson correlation coefficient, $r=0.79$ ) (Figure 1C). The activity of the low, medium and strong enhancers also showed significant difference $(\mathrm{p}<0.05$ and $\mathrm{p}<0.001$ for comparisons between different groups, Wilcoxon test) (Figure 1C).

\section{Enhancers are enriched within gene body}

STARR-seq identified enhancers in Drosophila genome are mostly located within genes and at promoter regions, especially in the introns (55.6\%). Only $22.6 \%$ are in intergenic regions. Drosophila enhancers are significantly underrepresented in repetitive sequences [16]. Different from Drosophila, a little more than $50 \%$ of enhancers are mapped to repetitive sequences related to TEs (Figure 2B) and nearly all of these enhancers $(4,831 / 5,020)$ contain repetitive elements including SINE, LINE, LTR, DNA transposones, satellite DNA and simple repeats (sTable 2). Different from animal genome, repetitive sequences in rice genome are gene-rich. The enrichment of enhancers in TE-related sequences in rice may be consistent with theories that TEs may regulate gene expression or even give rise to new genes during evolution [29, 30]. Identified enhancers are overrepresented in the 5' untranslated regions (5'UTR) and coding sequences, while obviously underrepresented in introns (Figure 2C), which are strikingly different from the distribution pattern of enhancers

144 in the Drosophila genome [16]. We divided the rice genome in two type, TE (repetitive sequences enriched with TEs) and non-TE (non-repetitive sequences) regions and analyzed the enhancer distribution relative to genes in these two types of 
sequences. Overall, the enhancer distribution patterns are quite similar in both TE and non-TE regions independent of the difference in gene activity in these two types of sequences (Figure 2D). Enhancers locate mostly within genes at the 5' prime and gradually drop to background level toward the 3' end of gene (Figure 2D).

\section{Proximal enhancers are absent from most genes in the rice genome}

Majority enhancers are mapped within or close to genes, which suggests that proximal regulation by enhancer may be a prevalent choice in the rice genome. Compared to the total number of annotated and predicted genes $(\sim 56,000)[31]$, the number of enhancers is relatively low $(9,642)$, less than 0.2 enhancer per gene averagely, suggesting most genes may be not directly regulated by enhancers in proximity (within TSS-5kb to TTS+5kb). Further analysis shows that $28.6 \%$ of genes $(15,997)$ (Figure 3A) with at least one enhancer in proximity, suggesting one enhancer may be in close distance to multiple genes.

We further separated genes into four groups according to their expression level (silent, low, medium and high) and calculated the percentage of genes with enhancer in proximity for each group. The percentage of genes with enhancer in proximity changes little for genes of different expression level in non-TE regions, and is slightly increased for genes in silence or expressed at low level in TE regions (Figure 3B). These results suggest STARR-seq enhancers at their endogenous loci are not enriched at actively transcribed genes, consistent with the episomal nature of the reporter plasmids.

\section{Genes in repetitive sequences are enriched with enhancers}

Repetitive sequences related to TEs are generally transcriptionally inactive, which make up $42.8 \%$ of the rice genome (Figure 2B) and harbors 15,839 (28.3\%) of total genes (Figure 3A, left panel). However, $52.1 \%$ of identified enhancers are located in TE regions (Figure 2B). These together suggest that genes in repetitive sequences are more enriched with enhancers. Actually, $39.6 \%$ of genes $(6,277)$ located in TE regions contain at least one enhancer in proximity (Figure 3A, left panel). 96.7\% of genes 
$177(15,318)$ in TE regions are of low expression level or silent and $40.1 \%$ of them $(6,141)$

178 contain at least one enhancer in proximity. For genes of higher transcription levels or

179 genes in non-TE regions, the percentage of genes with proximal enhancer is lower

180 than the average for total genes (Figure 3B, right panel). Gene ontology (GO)

181 analysis showed that genes in TE regions with enhancers in proximity occupy the top

18210 most significantly enriched categories, which are related to DNA replication,

183 integration, nucleotide binding, etc. (sFigure 7).

184

\section{Most identified enhancers are not DNase I hypersensitive sites (DHSs)}

DNase I hypersensitivity has been generally believed to be associated with sequences of activities, one possibility is transcription enhancing. $8.8 \%$ of all identified enhancers overlap with DHSs (Figure 4A). Genes with proximal enhancer overlapping with DHS in both TE and non-TE regions show higher expression level (Figure 4B). Similarly, only about $13.6 \%$ of enhancers identified in the human genome co-localize with DHSs (sTable 3) [20]. Differently, 48.5\% of enhancers identified in Drosophila co-localize with DHSs (sTable 3) [16]. The lack of DHS for the majority of enhancers proximal to actively transcribed genes may suggest that hypersensitivity to DNase I digestion may not be a universal mark for all functional enhancers in rice genome.

\section{H3K4me1 is not a ubiquitous enhancer mark in rice genome}

Histone modification mark H3K4me1 has been frequently used for enhancer prediction, which is enriched at enhancers identified by STARR-seq in both Drosophila and human genomes [16, 20]. However, for enhancers identified in rice, H3K4me1 is even less enriched than DHS, only $~ 330$ sites show strong signal of H3K4me1 (Figure 5A). H3K4me1 is nearly completely absent from predicted enhancers based on DHS locations in the genome (Figure 6A and sFigure 8).

\section{H3K4me3 is enriched at many identified enhancers}


207 portion of enhancers locate inside active genes at 5' UTR and in coding sequences

208

209

210

211

212

213

214

215

216

217

218

219

220

221

222

223

224

225

226

227

228

229

230

231

232

233

234

235

236

(Figure 2D and Figure 3). Consistently, H3K4me3 is found obviously enriched for enhancers identified, more obvious than any other histone mark or DHS (Figure 4C \& 4D, Figure 5A and sFigure 8).

\section{H3K27ac is an enriched enhancer mark}

Another histone modification mark frequently used for the prediction of active enhancer is $\mathrm{H} 3 \mathrm{~K} 27 \mathrm{ac}$, which has also been shown significantly enriched at enhancers identified by STARR-seq in both Drosophila and human genomes [16, 20]. Different from H3K4me1, H3K27ac is also enriched for identified enhancers in rice genome, the signal is especially strong for enhancers associated with DHS or located within non-TEs (red lines in Figure 4C \& 4D, Figure 5A and sFigure 8). Even enhancers not associated with DHS show slightly enrichment of H3K27ac (blue line in Figure 4C).

\section{H3K27me3 is enriched at a significant number of identified enhancers}

H3K27me3 is mostly associated with repressed genes. Surprisingly, we found that a significant number of identified enhancers are enriched with H3K27me3 (Figure 4C \& 4D), which is generally absent from enhancers identified in Drosophila and human genomes [16, 20]. Most of these enhancers are enriched strongly with H3K4me3 and H3K27ac as well (Figure 5A and sFigure 8). That whether these enhancers are poised or actively regulating genes is difficult to determine. Different from H3K27me3, $\mathrm{H} 3 \mathrm{~K} 9 \mathrm{me} 3$ is nearly completely absent from identified enhancers (Figure $\mathbf{5 A}$ and sFigure 8).

\section{Epigenetic clustering of identified enhancers}

We further grouped all enhancers into eight clusters based on the signal strength of multiple epigenetic marks including DHS, H3K4me1, H3K4me3, H3K27ac, H3K9me3 and H3K27me3 (Figure 5A and sFigure 8). Enhancers of cluster 1-7 (C1, sC2, C3-5, sC6 and C7; total 3,650 enhancers, 37.9\% of total enhancers identified; 
$237 \mathrm{sC2}$ and sC6 are unique for STARR-seq identified enhancers) are enriched with at

238 least one epigenetic mark (Figure 5A and sFigure 8). However, 62.1\% of enhancers

$239(5,992)$ are devoid of any analyzed epigenetic mark (C8, data not shown). H3K4me3

240 is strongly enriched for 4 clusters of enhancers which at the same time are enriched

241 with different levels of H3K27ac (Figure 5A and sFigure 8). Overall, H3K4me3 and

242 H3K27ac are mostly enriched, DHS and H3K27me3 are medially enriched,

243 H3K4me1 and H3K9me3 are least enriched for enhancers of C1-7 (Figure 5B \& 5C

244 and sFigure 8). About $17 \%$ and $83 \%$ of $\mathrm{C} 1-7$ enhancers locate inside TE and non-TE

245 regions, respectively (Figure 5D). Accordingly, majority of $\mathrm{C} 8$ enhancers (73\%) are

246 associated with TE regions (Figure 5D). Genes associated with each cluster of

247 enhancers are expressed at significantly ( $p<0.01$, Wilcoxon test) higher level in

248 non-TE regions than in TE regions except for cluster $7(\mathrm{p}=0.916)$ (Figure 5E).

249 Enhancers in cluster 7 are enriched with H3K27me3 (Figure 5A).

Predicted enhancers based on DHS location overlap little with STARR-seq identified enhancers

Enhancers had been predicted based on chromatin accessibility in Arabidopsis [23].

We followed the published methods and defined a DHS as enhancer if it locates beyond $1.5 \mathrm{~kb}$ upstream of TSS and at the same time is not in a gene body. By this method, 13,770 out of total 37,168 DHSs were predicted as enhancers (Figure 6A, sList 3). Only $20 \%$ of them are in TE regions and $80 \%$ of them are in non-TE regions (Figure 6B), consistent with the fact that the repetitive sequences are mostly in closed chromatin states with low accessibility. Due to the different distribution pattern, DHS predicted enhancers overlap with only a few STARR-seq enhancers (Figure 6C). Strikingly, clustering of predicted enhancers (DHS signal omitted and the C8 is different from STARR-seq enhancers $\mathrm{C} 8, \mathrm{dC} 2$ and $\mathrm{cC} 6$ are also unique) showed that even less of them are enriched with histone modification marks (Figure 6D). Overall, the expression of genes with proximal DHS predicted enhancer in TE and non-TE regions differ little ( $>0.05$, Wilcoxon test) except those being associated with 
8). Moreover, except dC6 and C8, genes with enhancers in proximity are not expressed significantly higher than the middle level of total genes (Figure 6E).

We further compared the distribution of STARR-seq enhancers, predicted DHS enhancers and other DHS sites (with DHS predicted enhancers excluded, non-predicted enhancer DHSs, sList 4). These three groups of elements show sharply different distribution patterns relative to the TSS of genes (Figure 6F). As previously shown, STARR-seq enhancers are mostly enriched within gene body favoring the 5' (Figure 6F, left). Most DHSs are closely positioned to TSSs. In fact, 91\% of all DHSs in rice genome are mapped within TSS+/-5kb regions, and similarly, $80.9 \%$ in Drosophila genome (sFigure 9). DHS predicted enhancers $(13,770$ out of total 37,168 DHSs genome-wide, 37\%) are at least $1.5 \mathrm{~kb}$ upstream away from TSS and out of gene body (Figure 6F, middle). For non-predicted enhancer DHSs $(23,398$ out of total $37,168,63 \%$ ) (sFigure 10a), they are predominantly enriched at upstream of TSS where promoters are located (Figure 6F, right), and only 1,347 of them overlap with STARR-seq enhancers (sFigure 10b). Due to close positioning to TSSs, significant portion of these DHSs overlap with sequences within the 200 bp upstream of TSS, +/-50bp of TSS and the 5' UTR regions (sFigure 10c), which is dramatically different from both DHS predicted and STARR-seq enhancers, respectively. Moreover, clustering on epigenetic modifications revealed that $50.5 \%$ of these sites are enriched with at least one type of modifications and most of them are enriched with H3K4me3, an active mark for active genes (sFigure 8, right). Though the majority of DHS sites are located not far from genes, these sites are less enriched inside gene body, where most of the identified enhancers are located.

\section{Discussion}

Enhancer prediction relies heavily on chromatin epigenetic mark. Though several histone modifications (H3K4me1 for most enhancers, H3K27ac for active enhancers, and coexistence of $\mathrm{H} 3 \mathrm{~K} 27 \mathrm{me} 3$ and $\mathrm{H} 3 \mathrm{~K} 4 \mathrm{me} 1$ for poised enhancers) have been 
297 frequently used for enhancer analysis, this method may fail to predict enhancers in

298 chromatin devoid of histone modifications. Moreover, enhancers predicted by

299 epigenetic marks are difficult to verify by genetic methods at large scale. To measure

300 enhancer activity of candidate sequences independent from chromatin context,

301 STARR-seq was invented [16] and had been successfully applied to the enhancer

302 analysis in both the Drosophila and human genomes [16, 20]. However, a

303 genome-wide functional analysis of enhancers for a plant genome has not been

304 reported, which severely limits our understanding of the nature and functional

305 mechanisms of plant enhancers.

Previous work predicting enhancers by chromatin sensitivity to DNase I digestion excluded arbitrarily DHSs within $1.5 \mathrm{~kb}$ upstream of TSS and in gene body [23]. In Drosophila, the majority of STARR-seq enhancers are actually located within gene body or in proximity to genes [16]. Similarly, our STARR-seq analysis of rice genome also shows that the majority of enhancers are localized within the gene body. The consistent observation of enhancer enrichment in the gene body in two evolutionarily far-separated genomes may suggest that most genes could be regulated by DNA elements built in their sequences (Figure 7). Furthermore, it will be interesting to see if enhancers in genes can also activate other genes separated by long distance. A capture Hi-C using identified enhancers as anchors may reveal if in plant genome genes separated far away can be co-regulated by enhancers located within the gene body.

Our analysis also reveals several unexpected features of identified enhancers in the rice genome. First, the majority of STARR-seq enhancers do not overlap with DHSs. DNase I hypersensitivity can be associated to any open and relaxed chromatin region, including insulators and other protein binding sites. Predicting enhancers relies solely on DHS location relative to the gene may fail to exclude DHSs which may possess non-enhancer activity. 
Second, though H3K4me1 has been used to predict enhancers in mammals, it is actually devoid from most STARR-seq enhancers and DHSs (independent of their location) in rice genome. The fact that $\mathrm{H} 3 \mathrm{~K} 4 \mathrm{me} 1$ is depleted from most DHSs further confirms that H3K4me1 may not be the ideal mark of enhancers in rice genome.

Third, many identified enhancers are enriched with H3K27me3. The majority of these enhancers are co-enriched with active chromatin marks of H3K4me3 and H3K27ac. Co-existence of H3K27me3 and H3K4me3 can be explained by the theory that many enhancers are poised. Surprisingly, H3K27ac and H3K27me3 co-exist at quite a few enhancer sites. These two modifications are supposed to be mutually exclusive. At this time, it is difficult to rule out possibilities that histones at these enhancers may be modified differently in different cells, or even differently on different allele in the same cell. In either case, it requires further careful analysis to reveal the underlying causes of this intriguing observation.

In summary, we presented a comprehensive enhancer activity map generated by quantitative measurement using STARR-seq for an important model plant. This method is especially suitable for functional analysis of small genomes. Successful characterization of enhancers in different cell types will help to improve our understanding of the nature of enhancers in the plant genome and sheds new lights into the elusive functional mechanisms of enhancers at large.

\section{Materials and methods}

\section{Screening vector}

For STARR-seq in rice cells, we constructed a screening vector based on the plasmid pPBI221 backbone and modified by introducing several sequences, which include a CMV 35S mini promoter, an intron and a GFP sequence, which are arranged sequentially and are underlined in the following DNA sequence. 
ggcttggggttagtctcaccagtcacagaaagcatcttacggatggcatgacagtaagagaattatgcagtgctgccataa ccatgagtgataacactgcggccaacttacttctgacaacgatcggaggaccgaaggagctaaccgcttttttgcacaaca tgggggatcatgtaactcgcettgatcgttgggaaccggagctgaatgaagccataccaaacgacgagcgtgacaccac gatgcctgtagcaatggcaacaacgttgcgcaaactattaactggcgaactacttactctagcttcccggcaacaattaata gactggatggaggcggataaagttgcaggaccacttctgcgctcggecettccggctggctggtttattgctgataaatct ggagccggtgagcgtgggtctcgcggtatcattgcagcactggggccagatggtaagccctcccgtatcgtagttatcta cacgacggggagtcaggcaactatggatgaacgaaatagacagatcgctgagataggtgcctcactgattaagcattgg taactgtcagaccaagtttactcatatatactttagattgatttaaaacttcatttttaatttaaaaggatctaggtgaagatcctttt tgataatctcatgaccaaaatccettaacgtgagttttcgttccactgagcgtcagacccegtagaaaagatcaaaggatctt cttgagatcctttttttctgcgcgtaatctgctgcttgcaaacaaaaaaaccaccgctaccagcggtggtttgtttgccggatc aagagctaccaactctttttccgaaggtaactggcttcagcagagcgcagataccaaatactgttcttctagtgtagccgta gttaggccaccacttcaagaactctgtagcaccgectacatacctcgetctgctaatcetgttaccagtggetgctgccagt ggcgataagtcgtgtcttaccgggttggactcaagacgatagttaccggataaggcgcagcggtcgggctgaacgggg ggttcgtgcacacagcccagcttggagcgaacgacctacaccgaactgagatacctacagcgtgagctatgagaaagc gccacgcttcccgaagggagaaaggcggacaggtatccggtaagcggcagggtcggaacaggagagcgcacgagg gagcttccagggggaaacgcctggtatctttatagtcctgtcgggtttcgccacctctgacttgagcgtcgatttttgtgatgc tcgtcaggggggcggagcctatggaaaaacgccagcaacgcggectttttacggttcctggecttttgctggecttttgctc acatgttctttcctgegttatccectgattctgtggataaccgtattaccgectttgagtgagctgataccgctcgecgcagcc gaacgaccgagcgcagcgagtcagtgagcgaggaagcggaagagcgcccaatacgcaaaccgcctctcccegcgc gttggccgattcattaatgcagctggcacgacaggtttccegactggaaagcgggcagtgagcgcaacgcaattaatgtg agttagctcactcattaggcacccaggctttacactttatgcttccggetcgtatgttgtgtggaattgtgagcggataacaa tttcacacaggaaacagctatgaccatgattacgccaagcttgggecgetcgagccgeccgggcgacttcgcaagacc cttcctctatataaggaagttcatttcatttggagagtccggatccattaacctgcaggtaaatttctagagctctggtag aaatctgagggtaaatttctagtttttctcettcattttcttggttaggaccettttctctttttatttttttgagctttgatct ttctttaaactgatctattttttaattgattggttatggtgtaaatattacatagctttaactgataatctgattactttat ttcgtgtgtctatgatgatgatgatagttacagaaccgacgactcgtcatgacatccactttgcetttctctccacagg tgtccactccaggtccaactgcaggtcgcetgcaggcttaaccatggctagcaaaggagaagaactcttcactg gagttgtcccaattcttgttgaattagatggtgatgtaaacggccacaagttctctgtcagtggagagggtgaaggt gatgcaacatacggaaaacttacctgaagttcatctgcactactggcaaactgcetgttccetggccaacactag tcactactctgtgctatggtgttcaatgcttttcaagatacccggatcatatgaaacggcatgactttttcaagagtg 
catgccegaaggttatgtccaggaaaggaccatcttcttcaaagatgacggcaactacaagacacgtgctgaag

tcaagtttgaaggtgataccettgttaatagaatcgagttaaaaggtattgacttcaaggaagatggcaacattctg

ggacacaaattggaatacaactataactcacacaatgtatacatcatggcagacaaacaaaagaatggaatcaa

agcgaacttcaagaccegccacaacattgaagatggaagcgttcaactagcagaccattatcaacaaaatactcc

aattggcgatggccetgtcettttaccagacaaccattacctgtccacacaatctgccetttcgaaagatccaacg

ctgatctagcgcatgcaccgggccetggtagaaatctgaggaaccgacgactcgtctgtacaacactctttccctacacg

acacacgtctgaactccagtctgtaattcacgcgtccagggatcgttcaaacatttggcaataaagtttcttaagattgaatcc

tgttgccggtcttgcgatgattatcatataatttctgttgaattacgttaagcatgtaataattaacatgtaatgcatgacgttattt

atgagatgggtttttatgattagagtcccgcaattatacatttaatacgcgatagaaaacaaaatatagcgcgcaaactagga

taaattatcgcgcgcggtgtcatctatgttactagatcgggccagtcgacccaagatcttccccggaattcactggccgtcg

ttttacaacgtcgtgactgggaaaaccetggcgttacccaacttaatcgccttgcagcacatccccetttcgccagctggcg

taatagcgaagaggccegcaccgatcgccttcccaacagttgcgcagcctgaatggcgaatggcgcetgatgcgtcta

gagtattttctccttacgcatctgtgcggtatttcacaccgcatatggtgcactctcagtacaatctgctctgatgccgcatagt

taagccagcccegacaccegccaacaccegctgacgcgecetgacgggettgtctgctcceggcatccgcttacagac

401

402

aagctgtgaccgtctccgggagctgcatgtgtcagaggtttcaccgtcatcaccgaaacgcgegagacgaaagggect

403

cgtgatacgcctatttttataggttaatgtcatgataataatggtttcttagacgtcaggtggcacttttcggggaaatgtgcgc

404 ggaacccctatttgtttatttttctaaatacattcaaatatgtatccgctcatgagacaataaccetgataaatgcttcaataatat tgaaaaaggaagagtatgagtattcaacatttccgtgtcgccettattccettttttgcggcattttgccttcctgttttgctcac ccagaaacgctggtgaaagtaaaagatgctgaagatcagttgggtgcacgagtgggttacatcgaactggatctcaaca gcggtaagatccttgagagtttcgccccgaagaacgttttccaatgatgagcacttttaaagttctgctatgtggcgcggta ttatcccgtattgacgccgggcaagagcaactcggtcgecgcatacactattctcagaatgacttggttgagtactcaccag tcacagaaaagcatcttacggatggcatgacagtaagagaattatgcagtgctgccataaccatgagtgataacactgcg

\section{Generation of input (screening) libraries}

412 We extracted genomic DNA from two weeks rice seedlings. About $125 \mu \mathrm{g}$ of genomic 413 DNA was diluted to $50 \mathrm{ng} / \mu \mathrm{l}$ and fragmented by sonication (Scientz II-D). DNA 414 fragments (500bp-800bp length) were size-selected, end repaired, 5'-phosphorylated 
and 3' dA-tailed with VAHTS Mate Pair Library Prep Kit for Illumina® (VAHTS; cat. no. ND104). VAHTS Adapters for Illumina was ligated to about $6 \mu \mathrm{g}$ of DNA fragments using VAHTS Mate Pair Library Prep Kit for Illumina® (VAHTS; cat. no. ND104) following the manufacturer's protocol. Adaptor ligated DNA was purified by using GeneJET PCR Purification Kit (Thermo Scientific; cat. no. K0702) then was amplified by TransStart FastPfu Fly DNA Polymerase (Transgen; cat. no. AP231) with Illumina sequencing primers (fw: ACACTCTTTCCCTACACGACG \& rev: GACTGGAGTTCAGACGTGTGC).

We obtained linear pPBI221 by PCR amplification $\left(95^{\circ} \mathrm{C}\right.$ for $5 \mathrm{~min}$; then 25 cycles of $95^{\circ} \mathrm{C}$ for $20 \mathrm{~s}, 57^{\circ} \mathrm{C}$ for $20 \mathrm{~s}$ and $72^{\circ} \mathrm{C}$ for $4 \mathrm{~min}$; Forward primer: ACACGTCTGAACTCCAGTCTGTAATTC \& Reverse primer: GTCGTGTAGGGAAAGAGTGTTGTACA). Circular plasmids was removed by digestion with DpnI (NEB; cat. no. R0176) at $37^{\circ} \mathrm{C}$ for $30 \mathrm{~min}$. PCR products were recovered with E.Z.N.A.® Gel Extraction Kit (Omega; cat. no. D2500). The adaptor ligated genomic DNA were recombined to linearized screening vector with ClonExprress II One Step Cloning Kit (Vazyme; cat. no. C112). For each reaction, 100ng linearized vector and 40ng adaptor ligated DNA was used in a total volume of

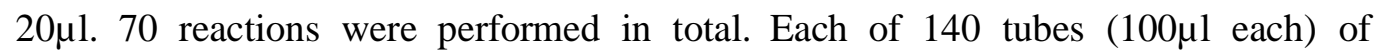
Trans1-T1 Phage Resistant Chemically Competent Cell (Transgen; cat. no. CD501) was transformed with $10 \mu \mathrm{l}$ of DNA, according to the manufacturer's protocol. 140 transformation reactions were pooled, transferred to $4 \mathrm{~L} \mathrm{LB}_{\mathrm{AMP}}$ medium, and grown to OD 0.8. Plasmids were purified using E.Z.N.A.® Endo-Free Plasmid Maxi Kit (Omega; cat. no. D6926) then quantified.

\section{Protoplast preparation}

The rice cultivar Nipponbare (Oryza sativa L. ssp japonica) seeds were moistened with water in Petri dishes at room temperature to promote germination (3days) and then were transferred to culture box at $37^{\circ} \mathrm{C}$ for about $24 \mathrm{~h}$, and then transplanted into soil. The rice plants were grown at $28^{\circ} \mathrm{C}$ with a relative humidity around $60 \%$. The 
445 light/dark photoperiod is $12 / 12 \mathrm{~h}$ by using a bank of $400 \mathrm{~W}$ metal halide lamps. The 446 light intensity is $400 \mu \mathrm{mol} \mathrm{m} \mathrm{s}^{-2}$ at the plant height. We collected 2-week-old rice 447 seedlings for the all experiments.

Protoplasts isolation is described below. Stem tissues from 80-120 rice seedlings were used. 5 seedlings were cut together into approximately $0.5 \mathrm{~mm}$ strips using sharp razors in $0.6 \mathrm{M}$ D-mannitol on Petri dishes. The strips were then digested $(1.5 \%$ Cellulase RS, 0.6 M mannitol, 0.1\% BSA, 0.75\% Macerozyme R-10, 3.4mM CaCl2, $10 \mathrm{mM}$ MES, $\mathrm{pH} 5.7$ ), and then vacuumed at $-50 \mathrm{kPa}$ in bottle for $30 \mathrm{~min}$. After that, the bottle was put on oscillator for $4-5 \mathrm{~h}$ in dark with gentle shaking (45rpm) at $28^{\circ} \mathrm{C}$. After digestion, protoplasts were filtered through 300 micron nylon meshes and washed 3-5 times with W5 solution (125 mM CaCl2, $154 \mathrm{mM} \mathrm{NaCl}, 5 \mathrm{mM} \mathrm{KCl}, 2$ $\mathrm{mM}$ MES, $\mathrm{pH}$ 5.7). Protoplasts were pelleted by centrifugation at $80 \mathrm{~g}$ for $5 \mathrm{~min}$. After another two wash with W5 solution (equal volume), protoplasts were then resuspended in MMG solution (15 mM MgCl2, 0.6M mannitol, $4 \mathrm{mM}$ MES, $\mathrm{pH}$ 5.7), and placed on ice for $30 \mathrm{~min}$. Protoplasts were centrifuged at $80 \mathrm{~g}$ again for $5 \mathrm{~min}$ and finally resuspended at a concentration of $1 \times 10^{7}$ cells per ml using the MMG solution, determined by using a hematocytometer. All procedures were carried out at room temperature.

\section{Plasmid library transformation}

For each sample, $30-40 \mu \mathrm{g}$ of plasmid was mixed with $100 \mu \mathrm{L}$ protoplasts $\left(\sim 1 \times 10^{6}\right.$

cells) in a $2 \mathrm{ml}$ tube with $110 \mu \mathrm{l}$ of freshly prepared PEG solution [40\% (W/V), $0.1 \mathrm{M}$ $\mathrm{CaCl}_{2}, 0.6 \mathrm{M}$ mannitol] added. Gently mix, then incubate in dark at $28^{\circ} \mathrm{C}$ for $15 \mathrm{~min}$. immediately at $80 \mathrm{~g}$ for $5 \mathrm{~min}$. The pelleted protoplasts were resuspended gently in $800 \mu \mathrm{l}$ of W5 solution. Finally, protoplasts transfected were cultured at $28^{\circ} \mathrm{C}$ for $16 \mathrm{~h}$

472 in dark. Reporter GFP signals were checked under fluorescent microscopy.

\section{Reporter cDNA libraries construction for Illumina sequencing}


$16 \mathrm{hrs}$ post-transfection, cells were washed in W5 solution twice and concentrated. Total RNA was extracted using TransZolTM Up Plus RNA Kit (ER501). polyA+ RNA fraction was isolated using VAHTS mRNA Capture Beads (N401). 5U DNase I (NEB M0303S) was used to digest DNA at $37^{\circ} \mathrm{C}$ for $20 \mathrm{~min}$.

Synthesis of first strand cDNA was carried out with TransScript One-Step gDNA Removal and cDNA synthesis SuperMix (AU311 $50^{\circ} \mathrm{C}$ for 30 min and $85^{\circ} \mathrm{C}$ for $5 \mathrm{~s}$ ) using a specific primer (GACTGGAGTTCAGACGTGTGC) and 8ul of RNA in 20 reactions. All reactions were pooled. The total amount of reporter cDNA was amplified for Illumina sequencing by a 2-step nested PCR strategy using the TransStart® FastPfu Fly DNA Polymerase (AP231-12).

In the first PCR $\left(98^{\circ} \mathrm{C}, 2 \mathrm{~min}\right.$; then $10-12$ cycles of $98^{\circ} \mathrm{C}$ for $30 \mathrm{~s}, 58^{\circ} \mathrm{C}$ for $30 \mathrm{~s}$, $72^{\circ} \mathrm{C}$ for $30 \mathrm{~s}$; and then $72^{\circ} \mathrm{C}$ for $5 \mathrm{~min}$ ), 10 -30ng cDNA were used for amplification with 2 reporter-specific primers (Fw: 5'GACTGGAGTTCAGACGTGTGC3' \& Rev: 5'GAGCTCTGGTAGAAATCTGAGGGTGTC3'), one of which spans the RNA splice junction of GFP intron. PCR products were purified by GeneJET PCR Purification Kit (K0701) and eluted in 20-30ul Elution Buffer (EB). Purified PCR product as template for the second PCR $\left(98^{\circ} \mathrm{C}\right.$ for $2 \mathrm{~min}$; followed by $6-10$ cycles of $98^{\circ} \mathrm{C}$ for $30 \mathrm{~s}, 65^{\circ} \mathrm{C}$ for $30 \mathrm{~s}, 72^{\circ} \mathrm{C}$ for $30 \mathrm{~s}$; and then $72{ }^{\circ} \mathrm{C}$ for $5 \mathrm{~min}$ ) with TransGen $\AA$ Biotech FastPfu Fly DNA Polymerase (AP231) and with VAHTS ${ }^{\mathrm{TM}}$ DNA Adapters for Illumina ${ }^{\circledR}(\mathrm{N} 302)$. PCR products were purified by GeneJET PCR Purification and eluted in 20-30 ul EB.

\section{Transfected plasmid library construction for Illumina sequencing}

When the PolyA RNA was capture, keep the total RNA (without mRNA) supernatant, add 10ul RNase A TransGen ${ }^{\circledR}$ Biotech GE $10120 \mathrm{mg} / \mathrm{ml}$ at $37^{\circ} \mathrm{C}$ for $30-60 \mathrm{~min}$, and the plasmid was purified by GeneJET PCR Purification Kit and eluted in 50 ul EB. Purified plasmid as template for the second PCR $\left(98^{\circ} \mathrm{C}\right.$ for $2 \mathrm{~min}$; followed by $8-12$ cycles of $98^{\circ} \mathrm{C}$ for $30 \mathrm{~s}, 65^{\circ} \mathrm{C}$ for $30 \mathrm{~s}, 72^{\circ} \mathrm{C}$ for $30 \mathrm{~s}$; and then $72{ }^{\circ} \mathrm{C}$ for $5 \mathrm{~min}$ ) with 
505 TransGen ${ }^{\circledR}$ Biotech FastPfu Fly DNA Polymerase and with VAHTS ${ }^{\mathrm{TM}}$ DNA Adapters

506 for Illumina ${ }^{\circledR}(\mathrm{N} 302)$. PCR products were purified by GeneJET PCR Purification and

507 eluted in 20-30 ul EB. All the libraries were sequenced on Illumine X Ten platform.

508

509 Computational analysis:

510 Mapping Illumina sequences

511 For 150mer paired-end reads generated by Illumina X Ten platform, bowtie2 [32] was

512 used to map them to the Nipponbare reference genome (IRGSP1.0). The default

513 parameters were used except parameter “--no-discordant -X 2000”. After mapping,

514 we used the samtools [33] to filter the mapped reads and kept only uniquely mapped

515 reads with parameter "view -bS -f 2 -q 5".

516

517 Computational processing of STARR-seq data

518 Unique fragments were used to compute the genome coverage in non-repeat region

519 and the enrichment of cDNA signal over plasmid input. Detailed procedures to

520 identify enhancers were described in Arnold et al (2013) [16]. And the program used

521 in this experiment was an $\mathrm{R}$ package named BasicSTARRseq. We performed

522 bonferroni correction to correct $\mathrm{p}$ values. Peaks are kept with $\mathrm{p}$-value smaller than

5230.001 and enrichment fold signal over 1.3. Only enhancers found in both replicates

524 were kept and used for further analysis. The overlapped enhancers were merged by

525 bedtools merge [34].

526

527 SATRR-seq enhancers overlap with DNase I hypersensitive sites (DHSs)

528 We consider it is an overlap if the middle point of an enhancer falls in a DHS site. By

529 this definition, 848 enhancers were found overlapping with DHS. DHS data were

530 generated by Zhang et al. [35]. DHS open sites were called by MACS1.4 [36] with

531 default parameter.

532

533 DHS predicted enhancers according to their location relative to genes

534 Enhancers were predicted in the rice genome following the definition published 
535 previously for enhancer prediction in Arabidopsis [23]. DHSs located in intergenic

536 regions (>1.5 kb upstream of TSS and downstream of transcription terminal site, TTS)

537 were arbitrarily defined as enhancers. Total 37,168 DHSs were called by MACS1.4 in

538 the whole rice genome. We used bedtools intersect to filter the DHSs in intergenic

539 regions and 13,770 DHSs were considered as predicted enhancers.

540

\section{Correlation analysis between different libraries}

542 Reads inside consecutive 500bp bins were used to calculate the correlation between

543 different libraries. Bins with value of zero were excluded from the calculation. We

544 used multiBamSummary and plotCorrelation in deeptools [37] with parameter:

545 --binSize 500 -ignoreDuplicates, and -corMethod pearson -skipZeros. Pearson

546 correlation coefficients were calculated.

\section{Coverage analysis}

549 Only unique fragment data were used to compute the read coverage at each position

550 for both repetitive and non-repetitive genome sequences. We used bedtools to

551 calculate the coverage ratio.

\section{Enhancer distribution in the genome}

554 We used MSU 7.0 to annotate the rice genome in the following categories: repeat, 555 coding sequence, core promoter (+/- 50 bp TSS around the TSS), 5' UTR, 3' UTR, 1st 556 intron, intron, 200bp upstream TSS, intergenic. Each enhancer was sorted into

557 different category if its summit falls within sequence of a specific category. The 558 distance between enhancer and proximal TSS was computed by bedtools closest 559 command.

\section{Analysis of GC-content effect of plasmid/cDNA libraries}

562 We calculated the GC content for each non-overlapping 100bp bins in the non-repeat 563 genome. Sequences were divided into 100 bins from $20 \%$ to $80 \%$ according to their 564 GC content. The cDNA/plasmid mean read coverage in each bin was also calculated. 
565 The softwares used are makewindows, nuc, coverage of bedtools suit.

566

567

568

569

570

571

572

573

574

575

576

577

578

579

580

581

582

583

584

585

586

587

588

589

590

591

592

593

594

\section{Intersecting genomic coordinates}

We used bedtools suite of programs to intersect genomic coordinates for STARR-seq enhancers, DHS enhancers and the closest TSS to a specific enhancer.

\section{STARR-seq, DHS-seq, and chromatin mark enrichment}

Histone modification data, including $\mathrm{H} 3 \mathrm{~K} 4 \mathrm{me} 3, \mathrm{H} 3 \mathrm{~K} 27 \mathrm{me} 3$ were retrieved from GSE19602 [38], and the H3K27ac, H3K9me3 were retrieved from GSE79033 [39], the H3K4me1 was retrieved from GSA under accession no. PRJCA000387 [40]. DHS data were from GSE26734 [35]. All the histone modifications were mapped to the reference genome IRGSP 1.0 using bowtie2 with parameter --no-discordant -X 2000 for paired-end data and --no-discordant for single-end data, and only the uniquely mapped reads were kept with duplicates removed by picardtools. The histone mark peaks were called by MACS1.4 with default parameters. Adapter was removed from the DHS sequencing data by cutadapt before mapping. Duplicates were removed with picardtools, and peaks were called by MACS1.4. Reads density at each position was normalized for comparison. 10,000 randomly selected regions of 700bp were used as control, and repeated for at least 10 times to calculate the mean value. The enrichment of chromatin mark and DHS were showed in a $5 \mathrm{~kb}$ window with the enhancer peak positioned in the center. STARR-seq enhancer, DHS enhancer and DHSs except predicted enhancers were divided into two groups depending on if they were located in repeat regions. STARR-seq enhancers were also divided into two groups according to their location relative to DHS. We used the R package EnrichedHeatmap [41] to plot the histone modifications (H3K4me1, H3k27ac, H3k4me3, H3K9me3, K3K27me3), and DHS enrichment for all groups with the center of analyzed elements positioned at middle point and extended to upstream and downstream by $5 \mathrm{~kb}$, respectively.

\section{Determine the proximity of enhancer to genes}


595 An enhancer is defined in proximity of a gene if it is located within the range of gene 596 body $+/-5 \mathrm{~kb}$ region. Genes were separated in two groups, TE and non-TE according 597 to their location in either TE or non-TE regions. The number of TE gene is 15,839 , 598 and the number of Non-TE gene is 39,961.The tools we used is BedTools. Gene 599 expression data are from Zhang et al. (2016) [31].

600

\section{GO analysis of genes with enhancer in proximity}

602

We used agriGO V2.0 [42] for the GO enrichment analysis.

603

\section{Histone modification at enhancers}

605 Relation between histone modifications, DHS and enhancers were analyzed. 3,650 out of total 9,642 STARR-seq enhancers overlap with at least one type of histone mark or DHS. 2,331 out of 13,770 DHS enhancers overlap with at least one kind of histone mark, while 11,819 out of 23,398 DHS peaks (with DHS enhancers excluded) overlap with at least one type of histone mark.

610

Enhancer categorizations based on epigenetic marks

Enhancers were classified into 8 clusters based on histone marks, DHS and relative enrichment of these marks. K-Means in Cluster3.0 [43] was used. Expression level of proximal genes with enhancers of each category was analyzed.

\section{Distribution of STARR-seq enhancers relative to the gene body}

TSSs and TTSs were aligned and sequences between were equally divided into 10 bins. Another $2.4 \mathrm{~kb}$ was extended upwards and downwards from TSS and TTS, respectively. The $2.4 \mathrm{~kb}$ extended regions were divided into 10 equal sized bins as well. The size of $2.4 \mathrm{~kb}$ was arbitrarily chosen for it is the median size of all genes in rice genome. 55,000 regions of $2.4 \mathrm{~kb}$ were randomly generate by bedtools random

622 command with parameter -n 55000 -1 2400, which were repeated for 100 times. Genes were separated in TE and non-TE groups. We counted the enhancer number in each 
626

627

628

629

630

631

632

633

634

635

636

637

638

639

640

641

642

643

644

645

646

647

648

649

650

651

652

653

random control.

\section{Percentage of genes with enhancers in proximity}

Genes were divided into four groups based on their expression level. High, RPKM >10; Medium, $1<$ RPKM $\leqq 10$; Low, $0<$ RPKM $\leqq 1$ and Silent. Genes were separated into two groups, TE and non-TE, to compare.

\section{Motif analysis}

For each group of elements, we submitted their sequences to the MEME-ChIP web server19 for de novo motif finding, using default parameters. The database we used was JASPAR CORE (2018) 20 plant database. Identified motifs are enclosed in supplementary List 5.

\section{Statistical analysis}

We used $\mathrm{R}$ for all statistical analysis.

\section{Data Availability}

All data are available under the accession number GSE121231.

\section{Authors' contributions}

JS constructed the reporter library and validated identified sites. LN designed and monitored the experiments. YZ participated in cell preparation, transfection and sequencing library preparation. NH carried out bioinformatics analysis. WS processed the raw data and participated in bioinformatics analysis. YZ helped with reporter library construction. LL advised on data analysis. $\mathrm{CH}$ designed, monitored the experiments and wrote the manuscript with input from all authors.

\section{Competing interests}

The authors have declared no competing interests. 


\section{Acknowledgements}

656 We gratefully acknowledge financial support from the National Natural Science 657 Foundation of China (31571347 to $\mathrm{CH}$ and 31771430 to LL), Guangdong Science and 658 Technology Department (2016A030313642 to CH), Shenzhen Science and 659 Technology Innovation Commission (JCYJ20150529152146478 to CH), Huazhong 660 Agricultural University Scientific \& Technological Self-innovation Foundation (to LL) 661 and the Young Thousand Talent Program (to $\mathrm{CH}$ ). We thank Dr. Shengtao Hou for 662 manuscript editing.

663

\section{References}

665 [1] Marand AP, Zhang T, Zhu B, Jiang J. Towards genome-wide prediction and 666 characterization of enhancers in plants. Biochim Biophys Acta Gene Regul Mech. $667 \quad 2017 ; 1860(1): 131-9$.

668 [2] Bulger M, Groudine M. Functional and mechanistic diversity of distal 669 transcription enhancers. Cell. 2011;144(3):327-39.

670 [3] Kieffer-Kwon KR, Tang Z, Mathe E, Qian J, Sung MH, Li G, et al. Interactome 671 maps of mouse gene regulatory domains reveal basic principles of transcriptional 672 regulation. Cell. 2013;155(7):1507-20.

673 [4] Li G, Ruan X, Auerbach RK, Sandhu KS, Zheng M, Wang P, et al. Extensive 674 promoter-centered chromatin interactions provide a topological basis for transcription 675 regulation. Cell. 2012;148(1-2):84-98.

676 [5] Tang Z, Luo OJ, Li X, Zheng M, Zhu JJ, Szalaj P, et al. CTCF-Mediated Human 677 3D Genome Architecture Reveals Chromatin Topology for Transcription. Cell. $678 \quad 2015 ; 163(7): 1611-27$.

679 [6] Whyte WA, Orlando DA, Hnisz D, Abraham BJ, Lin CY, Kagey MH, et al. Master 680 transcription factors and mediator establish super-enhancers at key cell identity genes. 681 Cell. 2013;153(2):307-19.

682 [7] Visel A, Taher L, Girgis H, May D, Golonzhka O, Hoch RV, et al. A 
683 high-resolution enhancer atlas of the developing telencephalon. Cell. $684 \quad 2013 ; 152(4): 895-908$.

685 1] Marand AP, Zhang T, Zhu B, Jiang J. Towards genome-wide prediction and 686 characterization of enhancers in plants. Biochim Biophys Acta Gene Regul Mech. $687 \quad 2017 ; 1860(1): 131-9$.

688 [2] Bulger M, Groudine M. Functional and mechanistic diversity of distal 689 transcription enhancers. Cell. 2011;144(3):327-39.

690 [3] Kieffer-Kwon KR, Tang Z, Mathe E, Qian J, Sung MH, Li G, et al. Interactome 691 maps of mouse gene regulatory domains reveal basic principles of transcriptional 692 regulation. Cell. 2013;155(7):1507-20.

693 [4] Li G, Ruan X, Auerbach RK, Sandhu KS, Zheng M, Wang P, et al. Extensive 694 promoter-centered chromatin interactions provide a topological basis for transcription 695 regulation. Cell. 2012;148(1-2):84-98.

696 [5] Tang Z, Luo OJ, Li X, Zheng M, Zhu JJ, Szalaj P, et al. CTCF-Mediated Human 697 3D Genome Architecture Reveals Chromatin Topology for Transcription. Cell. $698 \quad 2015 ; 163(7): 1611-27$.

699 [6] Whyte WA, Orlando DA, Hnisz D, Abraham BJ, Lin CY, Kagey MH, et al. 700 Master transcription factors and mediator establish super-enhancers at key cell 701 identity genes. Cell. 2013;153(2):307-19.

702 [7] Visel A, Taher L, Girgis H, May D, Golonzhka O, Hoch RV, et al. A 703 high-resolution enhancer atlas of the developing telencephalon. Cell. $704 \quad 2013 ; 152(4): 895-908$.

705 [8] Hnisz D, Abraham BJ, Lee TI, Lau A, Saint-Andre V, Sigova AA, et al. 706 Super-enhancers in the control of cell identity and disease. Cell. 2013;155(4):934-47.

707 [9] Zentner GE, Tesar PJ, Scacheri PC. Epigenetic signatures distinguish multiple 708 classes of enhancers with distinct cellular functions. Genome Res. 709 2011;21(8):1273-83.

710 [10] Kim TK, Hemberg M, Gray JM, Costa AM, Bear DM, Wu J, et al. Widespread 711 transcription at neuronal activity-regulated enhancers. Nature. 2010;465(7295):182-7. 712 [11] Creyghton MP, Cheng AW, Welstead GG, Kooistra T, Carey BW, Steine EJ, et al. 
713 Histone H3K27ac separates active from poised enhancers and predicts developmental 714 state. Proc Natl Acad Sci U S A. 2010;107(50):21931-6.

715 [12] Visel A, Blow MJ, Li Z, Zhang T, Akiyama JA, Holt A, et al. ChIP-seq accurately 716 predicts tissue-specific activity of enhancers. Nature. 2009;457(7231):854-8.

717 [13] Heintzman ND, Hon GC, Hawkins RD, Kheradpour P, Stark A, Harp LF, et al. 718 Histone modifications at human enhancers reflect global cell-type-specific gene 719 expression. Nature. 2009;459(7243):108-12.

720 [14] Heintzman ND, Stuart RK, Hon G, Fu Y, Ching CW, Hawkins RD, et al. Distinct 721 and predictive chromatin signatures of transcriptional promoters and enhancers in the 722 human genome. Nat Genet. 2007;39(3):311-8.

723 [15] Visel A, Rubin EM, Pennacchio LA. Genomic views of distant-acting enhancers. $724 \quad$ Nature. 2009;461(7261):199-205.

725 [16] Arnold CD, Gerlach D, Stelzer C, Boryn LM, Rath M, Stark A. Genome-wide 726 quantitative enhancer activity maps identified by STARR-seq. Science. $727 \quad 2013 ; 339(6123): 1074-7$.

728 [17] Inoue F, Ahituv N. Decoding enhancers using massively parallel reporter assays. 729 Genomics. 2015;106(3):159-64.

730 [18] Vanhille L, Griffon A, Maqbool MA, Zacarias-Cabeza J, Dao LT, Fernandez N, et 731 al. High-throughput and quantitative assessment of enhancer activity in mammals by 732 CapStarr-seq. Nat Commun. 2015;6:6905.

733 [19] Dao LTM, Galindo-Albarran AO, Castro-Mondragon JA, Andrieu-Soler C, 734 Medina-Rivera A, Souaid C, et al. Genome-wide characterization of mammalian 735 promoters with distal enhancer functions. Nat Genet. 2017;49(7):1073-81.

736 [20] Liu Y, Yu S, Dhiman VK, Brunetti T, Eckart H, White KP. Functional assessment 737 of human enhancer activities using whole-genome STARR-sequencing. Genome Biol. $738 \quad 2017 ; 18(1): 219$.

739 [21] Muerdter F, Boryn LM, Woodfin AR, Neumayr C, Rath M, Zabidi MA, et al. 740 Resolving systematic errors in widely used enhancer activity assays in human cells. 741 Nat Methods. 2018;15(2):141-9.

742 [22] Liu S, Liu Y, Zhang Q, Wu J, Liang J, Yu S, et al. Systematic identification of 
743 regulatory variants associated with cancer risk. Genome Biol. 2017;18(1):194.

744 [23] Zhu B, Zhang W, Zhang T, Liu B, Jiang J. Genome-Wide Prediction and

745 Validation of Intergenic Enhancers in Arabidopsis Using Open Chromatin Signatures.

$746 \quad$ Plant Cell. 2015;27(9):2415-26.

747 [24] Yang W, Jefferson RA, Huttner E, Moore JM, Gagliano WB, Grossniklaus U. An

748 egg apparatus-specific enhancer of Arabidopsis, identified by enhancer detection.

749 Plant Physiol. 2005;139(3):1421-32.

750 [25] Clark RM, Wagler TN, Quijada P, Doebley J. A distant upstream enhancer at the 751 maize domestication gene tb1 has pleiotropic effects on plant and inflorescent 752 architecture. Nat Genet. 2006;38(5):594-7.

753 [26] McGarry RC, Ayre BG. A DNA element between At4g28630 and At4g28640

754 confers companion-cell specific expression following the sink-to-source transition in 755 mature minor vein phloem. Planta. 2008;228(5):839-49.

756 [27] Schauer SE, Schluter PM, Baskar R, Gheyselinck J, Bolanos A, Curtis MD, et al. 757 Intronic regulatory elements determine the divergent expression patterns of 758 AGAMOUS-LIKE6 subfamily members in Arabidopsis. Plant J. $7592009 ; 59(6): 987-1000$.

760 [28] Raatz B, Eicker A, Schmitz G, Fuss E, Muller D, Rossmann S, et al. Specific 761 expression of LATERAL SUPPRESSOR is controlled by an evolutionarily conserved 762 3' enhancer. Plant J. 2011;68(3):400-12.

763 [29] Zhao D, Ferguson AA, Jiang N. What makes up plant genomes: The vanishing 764 line between transposable elements and genes. Biochim Biophys Acta. $765 \quad 2016 ; 1859(2): 366-80$.

766 [30] Hirsch CD, Springer NM. Transposable element influences on gene expression in 767 plants. Biochim Biophys Acta Gene Regul Mech. 2017;1860(1):157-65.

768 [31] Zhang J, Luo W, Zhao Y, Xu Y, Song S, Chong K. Comparative metabolomic 769 analysis reveals a reactive oxygen species-dominated dynamic model underlying 770 chilling environment adaptation and tolerance in rice. New Phytol. $771 \quad 2016 ; 211(4): 1295-310$.

772 [32] Langmead B, Salzberg SL. Fast gapped-read alignment with Bowtie 2. Nat 
Methods. 2012;9(4):357-9.

[33] Li H, Handsaker B, Wysoker A, Fennell T, Ruan J, Homer N, et al. The Sequence Alignment/Map format and SAMtools. Bioinformatics. 2009;25(16):2078-9.

[34] Quinlan AR, Hall IM. BEDTools: a flexible suite of utilities for comparing genomic features. Bioinformatics. 2010;26(6):841-2.

[35] Zhang W, Wu Y, Schnable JC, Zeng Z, Freeling M, Crawford GE, et al. High-resolution mapping of open chromatin in the rice genome. Genome Res. 2012;22(1):151-62.

[36] Zhang Y, Liu T, Meyer CA, Eeckhoute J, Johnson DS, Bernstein BE, et al. Model-based analysis of ChIP-Seq (MACS). Genome Biol. 2008;9(9):R137.

[37] Ramirez F, Ryan DP, Gruning B, Bhardwaj V, Kilpert F, Richter AS, et al. deepTools2: a next generation web server for deep-sequencing data analysis. Nucleic Acids Res. 2016;44(W1):W160-5.

[38] He G, Zhu X, Elling AA, Chen L, Wang X, Guo L, et al. Global epigenetic and transcriptional trends among two rice subspecies and their reciprocal hybrids. Plant Cell. 2010;22(1):17-33.

[39] Fang Y, Wang X, Wang L, Pan X, Xiao J, Wang XE, et al. Functional characterization of open chromatin in bidirectional promoters of rice. Sci Rep. 2016;6:32088.

[40] Pan X, Fang Y, Yang X, Zheng D, Chen L, Wang L, et al. Chromatin states responsible for the regulation of differentially expressed genes under (60)Co gamma ray radiation in rice. BMC Genomics. 2017;18(1):778.

[41] Gu Z, Eils R, Schlesner M, Ishaque N. EnrichedHeatmap: an R/Bioconductor package for comprehensive visualization of genomic signal associations. BMC Genomics. 2018;19(1):234.

[42] Tian T, Liu Y, Yan H, You Q, Yi X, Du Z, et al. agriGO v2.0: a GO analysis toolkit for the agricultural community, 2017 update. Nucleic Acids Res. 2017;45(W1):W122-W9.

[43] de Hoon MJ, Imoto S, Nolan J, Miyano S. Open source clustering software. Bioinformatics. 2004;20(9):1453-4. 
803

804

805

806

807

808

809

810

811

812

813

814

815

816

817

818

819

820

821

822

823

824

825

826

827

828

829

830

831

\section{Figure legends}

\section{Figure 1 Genome-wide quantitative enhancer discovery}

A. STARR-seq work flow. CMV 35s mini promoter was used to drive basal expression of reporter gene [ORF, open-reading frame; pA site, polyadenylation site].

B. STARR-seq cDNA (red) and input plasmid (grey) fragment densities in a genomic locus. Black boxes denote identified enhancers in two replicates. C. STARR-seq and Real-time PCR quantification are linearly correlated: r, Pearson correlation coefficient; Error bars indicate two independent biological replicates; Inset, the same data depicted in box plot; $* \mathrm{p}<0.05, * * * \mathrm{p}<0.001$, Wilcoxon rank-sum test.

\section{Figure 2 Genomic distribution of STARR-seq identified enhancers}

A. Enhancers identified by two independent STARR-seq experiments. 9,642 enhancers were discovered in both replicates. B and C. Distribution and relative enrichment of identified enhancers in the rice genome. D. Distribution of enhancers relative to the gene body. TSS (transcription start site) and TTS (transcription termination site) are aligned and gene bodies are divided into 10 equal bins. Extended from TSS to upstream and from TTS to downstream are 10 bins of equal size (240bp, determined by dividing the median gene size by 10$)$, respectively.

Figure 3 Presence of identified enhancers in proximity to genes of different expression level

A. Number of genes expressed at different levels with or without enhancers in proximity in TE (left) or non-TE regions (right), respectively. Enhancers within the range from TSS $-5 \mathrm{~kb}$ to TTS $+5 \mathrm{~kb}$ are defined as in proximity to genes. B. Percentage of genes expressed at different levels in TE (left) or non-TE regions (right) with or without enhancers in proximity. Silent, RPKM=0; Low, $0<\mathrm{RPKM} \leqq 1$; Medium, $1<$ RPKM $\leqq 10 ;$ High, RPKM $>10$. 
Figure 4 Epigenetic characteristics of identified enhancers at their endogenous

loci

A. Numbers of identified enhancers overlap with DNase I hypersensitive sites, DHSs.

B. Expression of genes in TE or non-TE regions with proximal enhancers overlapping enrichment for enhancers overlapping with DHSs (red) or not (blue). D. Epigenetic signal enrichment for enhancers in non-TE (red) and TE (blue) regions, respectively.

\section{Figure 5 Epigenetically clustered STARR-seq enhancers}

A. Identified enhancers are grouped into 8 clusters based on the signal strength of 6 epigenetic marks. Numbers of enhancers in each cluster are shown at the bottom. Overall signal density was ranked: absent (close to background level) as light grey box, low as light red, medium as dark red and high as bright red. B. Percentage of identified enhancers in each cluster with (red) or without (light grey) indicated epigenetic mark. C. Identified enhancers with indicated epigenetic mark in each cluster were further divided into three sub-classes based on the average signal density, percentage of enhancers of low, medium and high signal density are shown. D. Percentage of cluster 1-7 and C8 in TE (light grey) or non-TE (red) regions. E. Expression of genes in TE (blue) or non-TE (red) regions with enhancers of different cluster in proximity. Genes in non-TE regions with enhancers of nearly all clusters (except $\mathrm{C} 7)$ show significant $(\mathrm{p}<0.01)$ higher expression level than in TEs.

\section{Figure 6 Predicted enhancers based on DHSs location to genes}

A. Numbers of predicted enhancers $(13,770)$ according to DHSs location and of DHSs in the genome. C. Overlap of predicted enhancers and STARR-seq enhancers. D. Pie charts of clustered enhancers. For predicted enhancers, DHS signal was omitted for clustering. E. Expression of genes in TE (blue) or non-TE (red) regions with 
862

863

864

865

866

867

868

869

870

871

872

873

874

875

876

877

878

879

880

881

882

883

884

885

886

887

888

889

890

regions. F. Relative enrichment of identified enhancers, predicted enhancers and DHS sites excluding predicted enhancers around the TSS. Red and blue lines show sites in non-TE or TE regions, respectively.

Figure 7 Proposed enhancer function model in rice genome. Gene body is shown in green. Density of identified enhancers gradually decreases within gene body from 5, TSS to the 3' TTS. Genes can be self-regulated by enhancers located within. Enhancers in genes may also activate other genes separated far away. Predicted enhancers are mostly located away from genes and if they activate genes or not requires systematic experimental validation.

\section{Supplementary materials}

Figures $\mathrm{S} 1$ to $\mathrm{S} 10$

Tables S1 to S3

Lists $\mathrm{S} 1$ to $\mathrm{S} 5$

\section{Supplementary Figure 1 Plasmid and cDNA libraries}

A and B. Fragment sizes of STARR-seq input (top left) and cDNA libraries (top right), the median fragment sizes are indicated. C and D. Coverage of the rice non-repetitive genome by independent fragments (cumulative), $\mathbf{C}$ for plasmid library, $\mathbf{D}$ for cDNA library. $\mathbf{E}$ and $\mathbf{F}$. Coverage of the rice repetitive genome by independent fragments (cumulative), $\mathbf{E}$ for plasmid library, $\mathbf{F}$ for cDNA library. More than $90 \%$ of the genome is covered by more than one fragment in input plasmid library.

\section{Supplementary Figure 2 GC content analysis for plasmid and cDNA libraries}

The genome sequence was binned according to GC-content (x axis). Each boxplot shows the read depth of all positions within the respective windows, revealing that STARR-seq input shows similar pattern as the cDNA library. 
891 Supplementary Figure 3 Correlation analysis of all sequenced libraries. See

892 Methods for analysis description.

893

894

Supplementary Figure 4 Correlation analysis of enhancers from two replicates

895 For all enhancers identified by two independent biological replicates, the correlation

896 of their activity was reasonably high with a Pearson correlation coefficient $r=0.604$.

897

898

Supplementary Figure 5 Enhancer distribution relative to their strength

899

900

Supplementary Figure 6 Number of unique fragments supporting enhancer

901 identification

902 Percentage of enhancers versus the number of independent genomic fragments per 903 enhancer.

904

905

\section{Supplementary Figure 7 GO analysis of genes mostly enriched}

906

Top ten categories of enriched genes with identified enhancer in proximity. Most of

907 them are located within TE regions and are involved in the biological process of DNA

908 synthesis.

909

Supplementary Figure 8 Enhancer and DHSs clustering on epigenetic signals

911 STARR-seq enhancers, DHS predicted enhancers and the remaining DHSs are clustered. Note that the two groups of DHSs were clustered without the values of DNase I hypersensitivity. sC2, sC6 are unique for STARR-seq enhancers. dC2, dC6 are unique for the two groups of DHS elements.

915

\section{Supplementary Figure 9 Distribution of DHSs relative to TSSs}

917 The relative DHSs enrichment in Drosophila and rice genomes are calculated in

918 moving 500bp bins normalized against total number of bins at each position. 0 on $\mathrm{X}$ 919 axis corresponding to TSS. 


\author{
921 Supplementary Figure 10 DHSs with predicted enhancer sites excluded \\ 922 A. Total DHSs are divided into two groups, 13,770 sites as predicted enhancers, \\ 92323,398 sites within TSS-1.5kb and in gene body. B. Overlap of STARR-seq enhancers \\ 924 and non-predicted enhancer DHSs sites. C. Relative enrichment of non-predicted \\ 925 enhancer DHSs sites in different categories of genome sequences. \\ 926 \\ 927 Supplementary Table 1 Sequencing statistics of libraries \\ 928 \\ 929 Supplementary Table 2 STARR-seq enhancers in TE regions overlap with \\ 930 different types of repetitive sequences \\ 931 \\ 932 Supplementary Table 3 Percentage of STARR-seq enhancers overlapping with \\ 933 DHS \\ 934 \\ 935 \\ Supplementary List 1 STARR-seq identified enhancers \\ 936 \\ 937 Supplementary List 2 Real-Time PCR validated enhancer sites \\ 938 \\ 939 Supplementary List 3 Predicted enhancers according to DHS location \\ 940 \\ 941 Supplementary List 4 non-predicted-enhancer DHSs \\ 942 \\ 943 Supplementary List 5 Identified motifs for different groups of elements
}


A

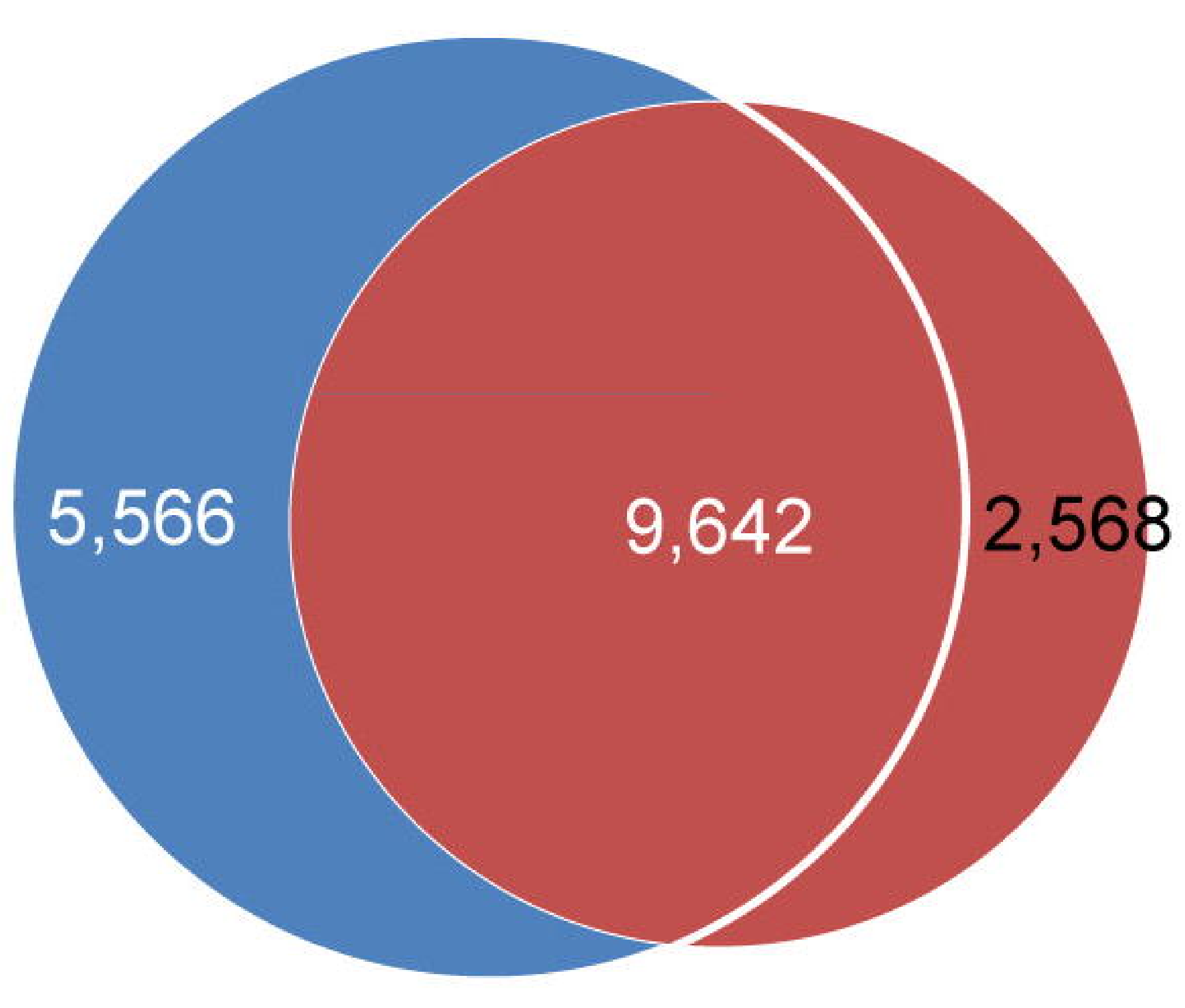

C

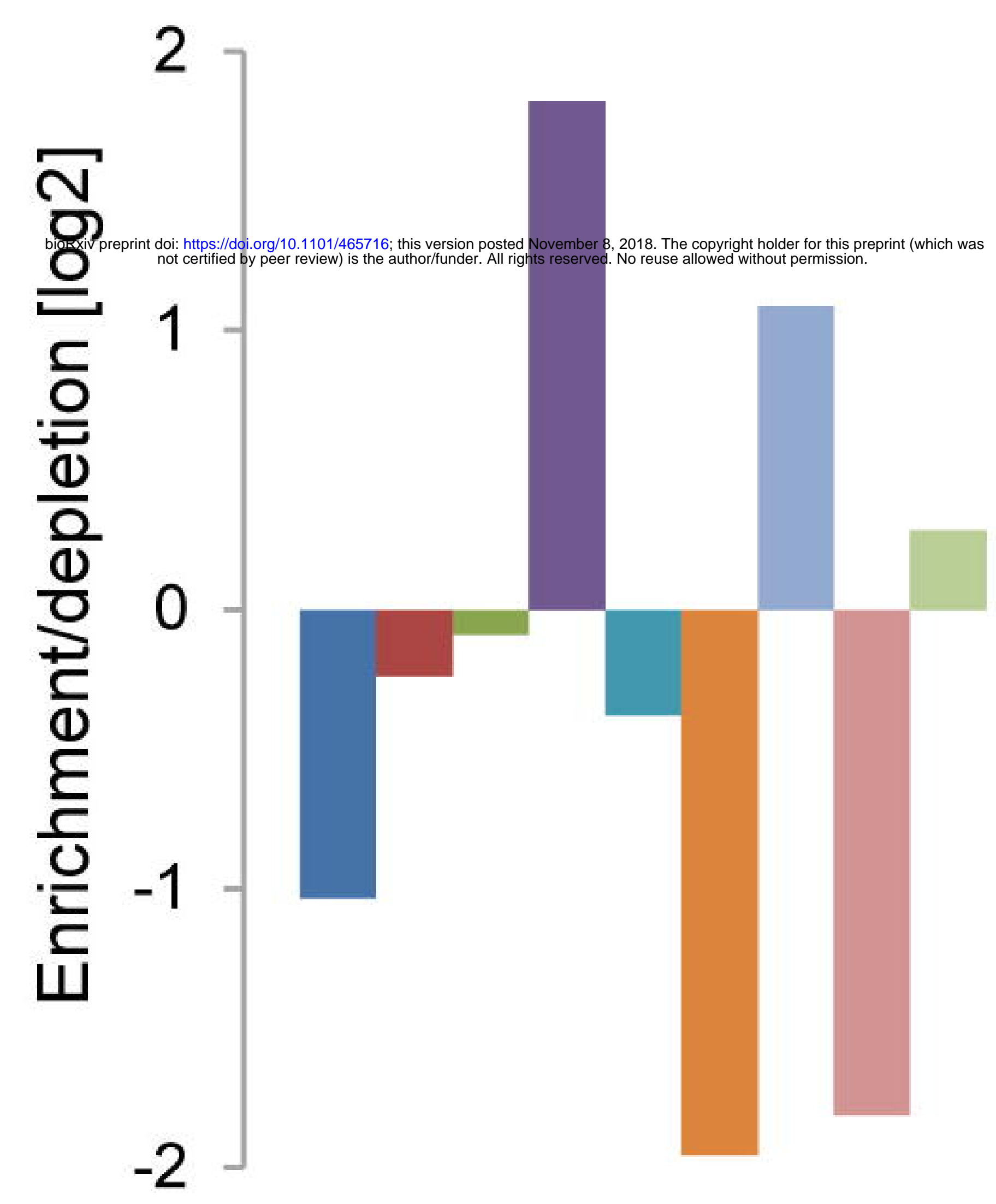

- intergenic

- intron

- 200bp upstream TSS $₫$ Coding sequence

$+/-50$ bp TSS

- 5'UTR

1st intron

3'UTR

repeats
Genome

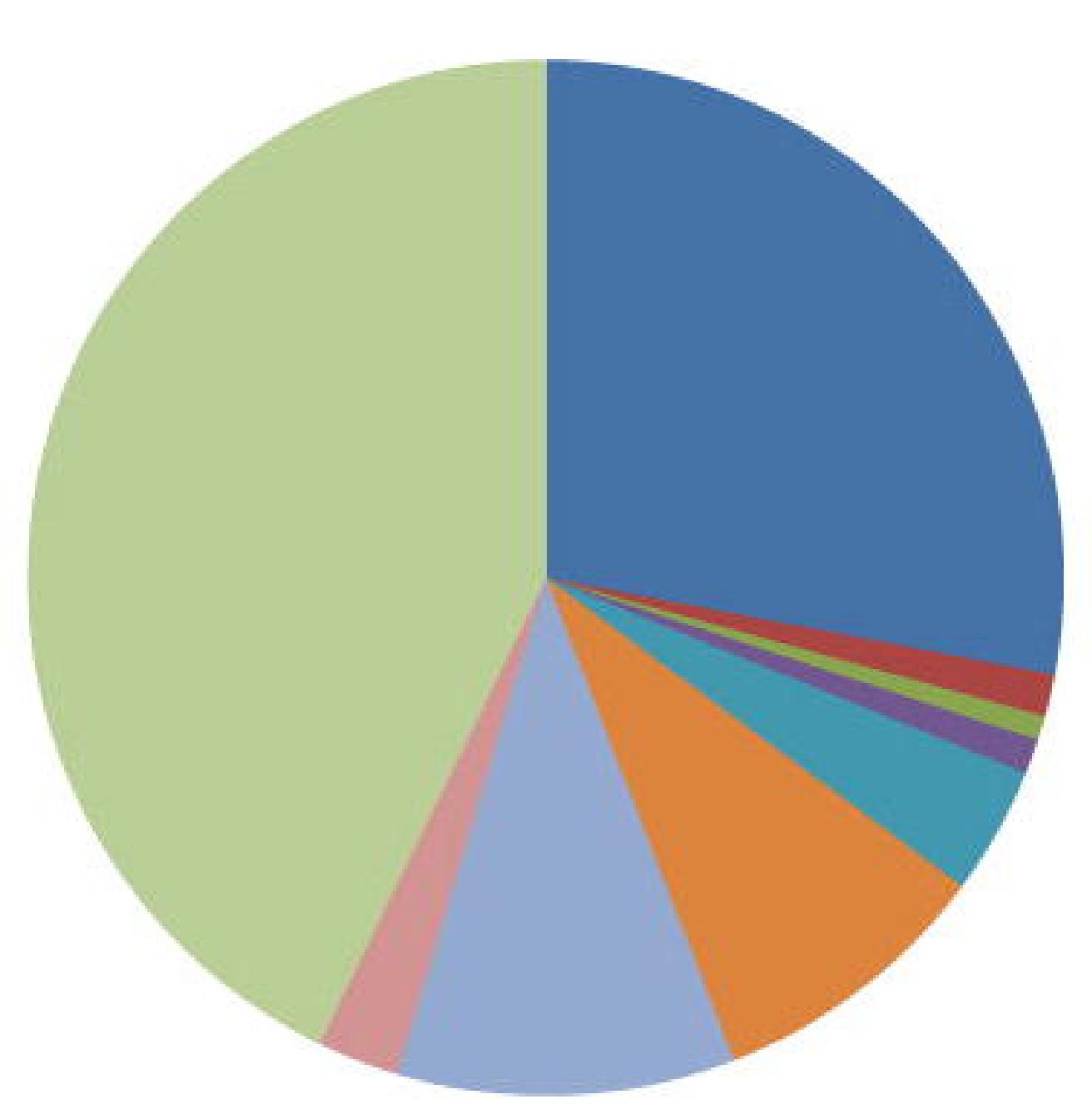

- intergenic

- 200bp upstream TSS

$+/-50$ bp TSS

D
STARR-seq Enhancers

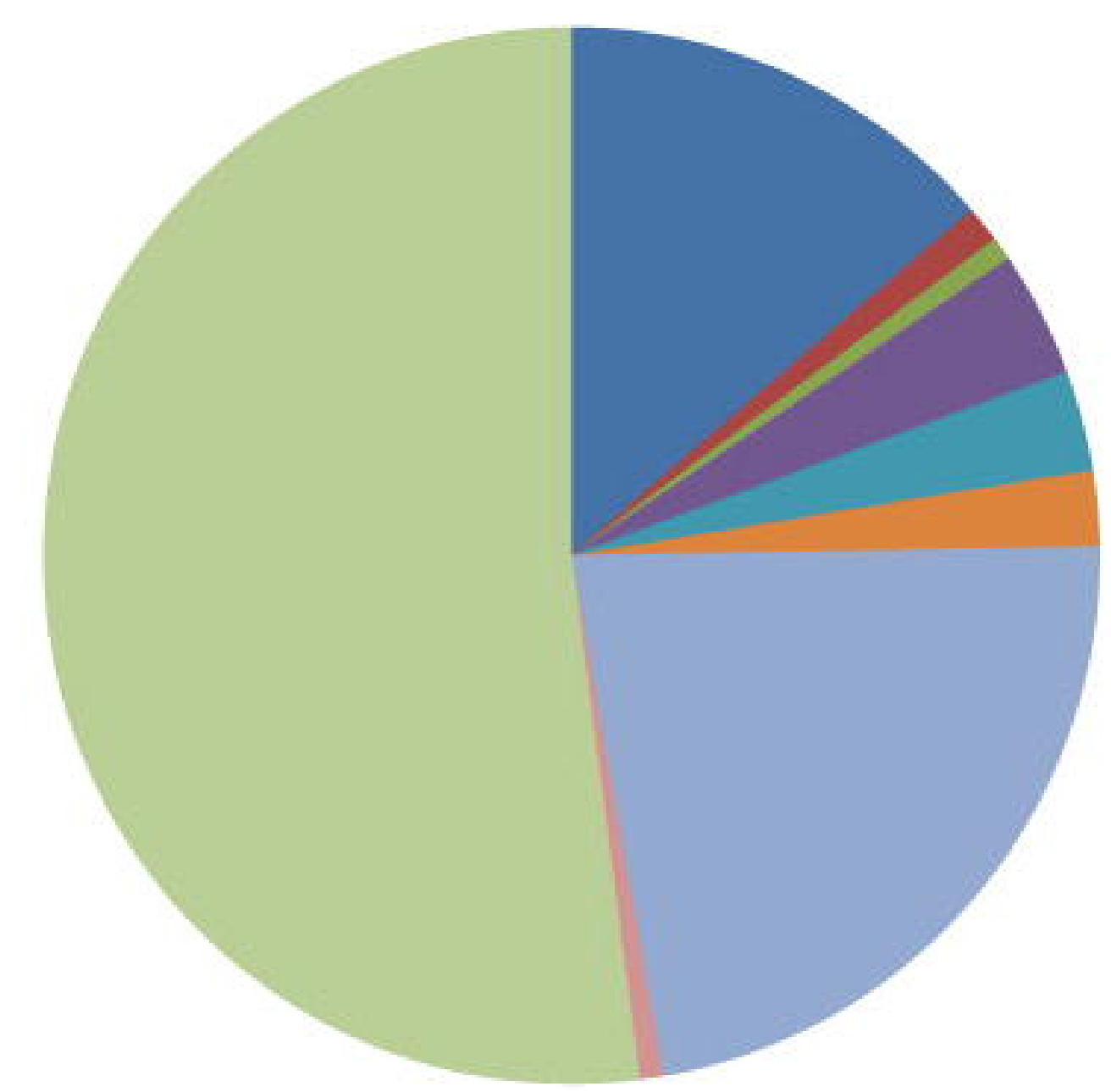

-5'UTR $\quad$ Coding sequence

-1st intron $\quad$ 3'UTR

mintron $\quad$ repeats

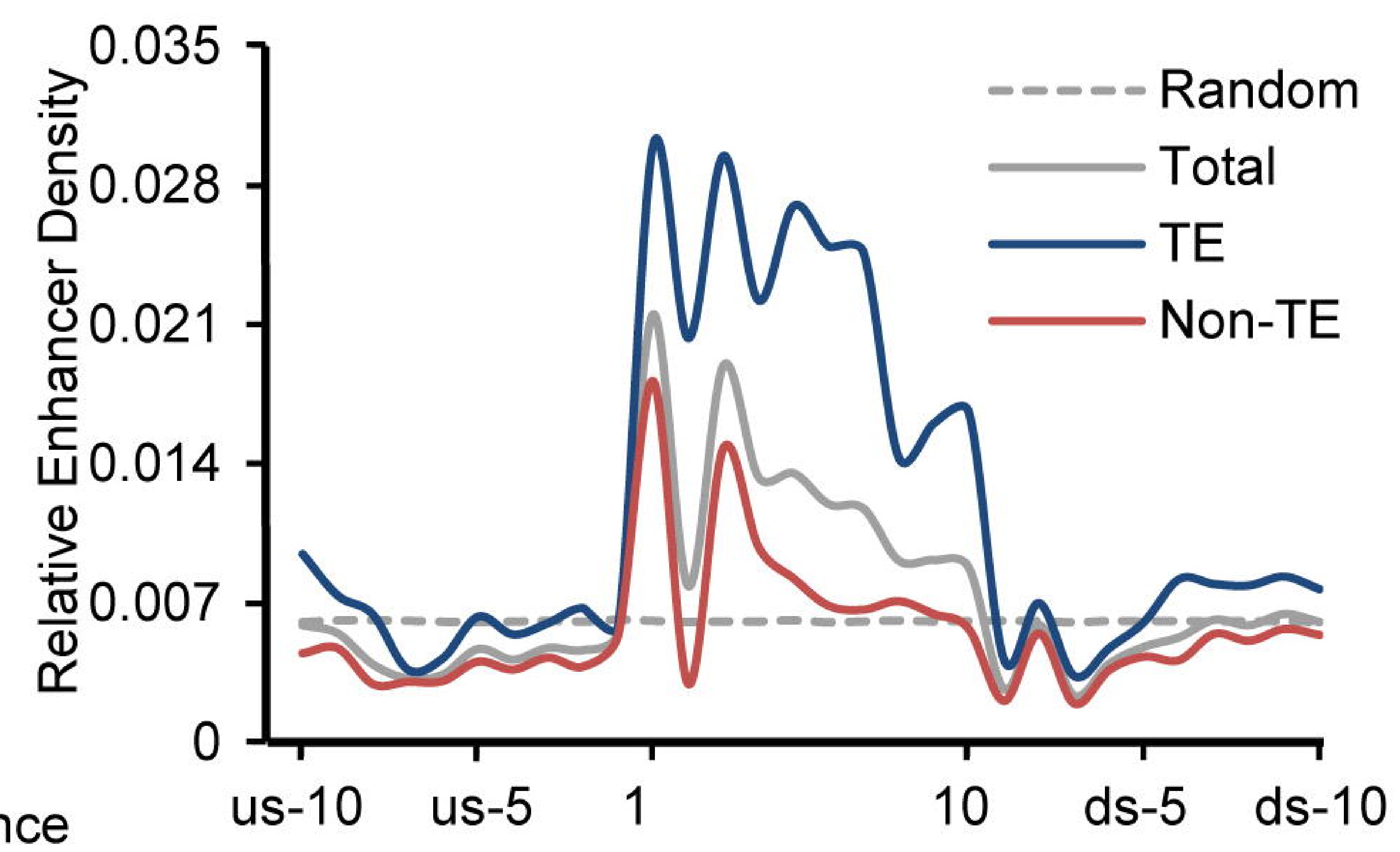


Figure 3, Sun et al.

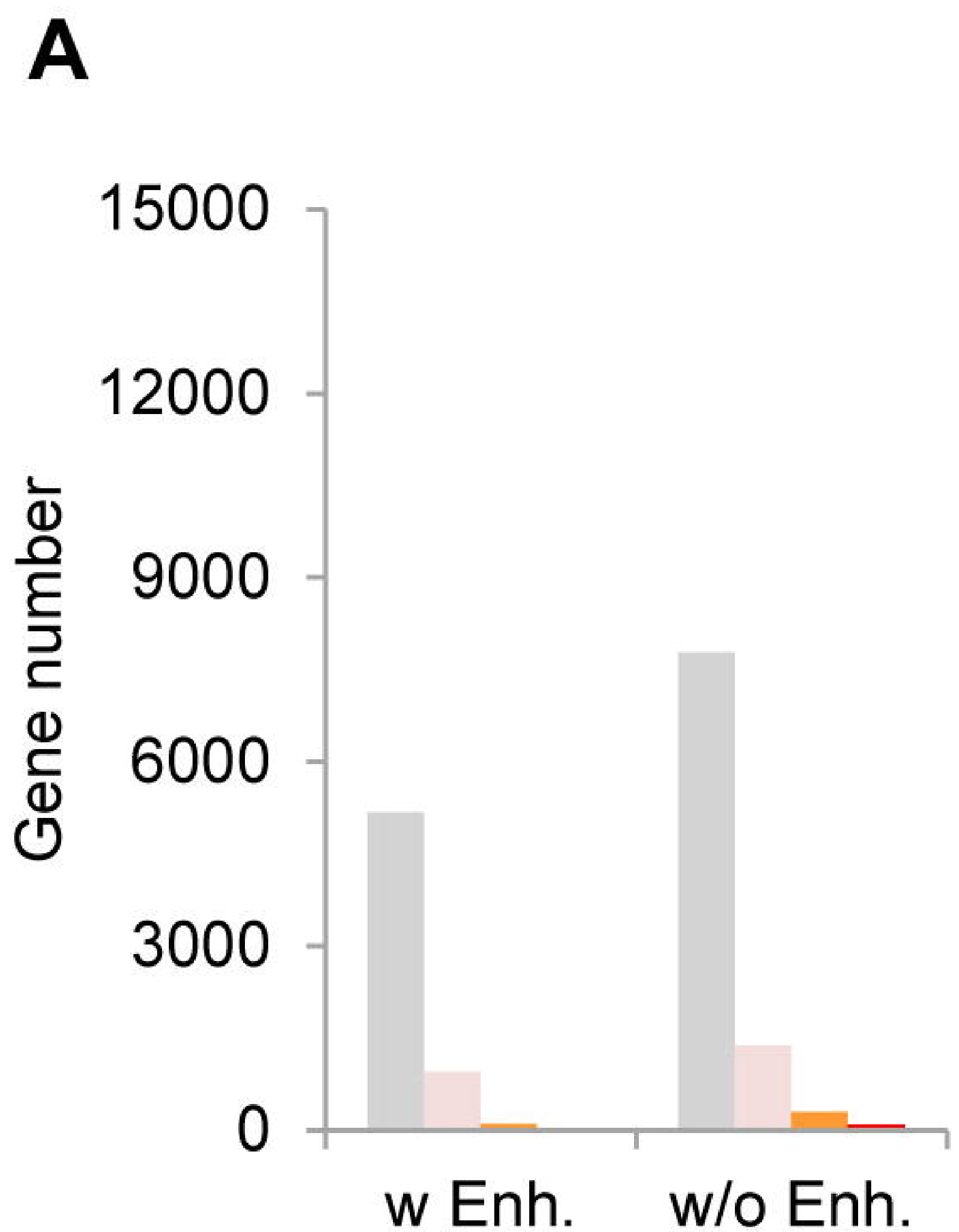

Genes in TE
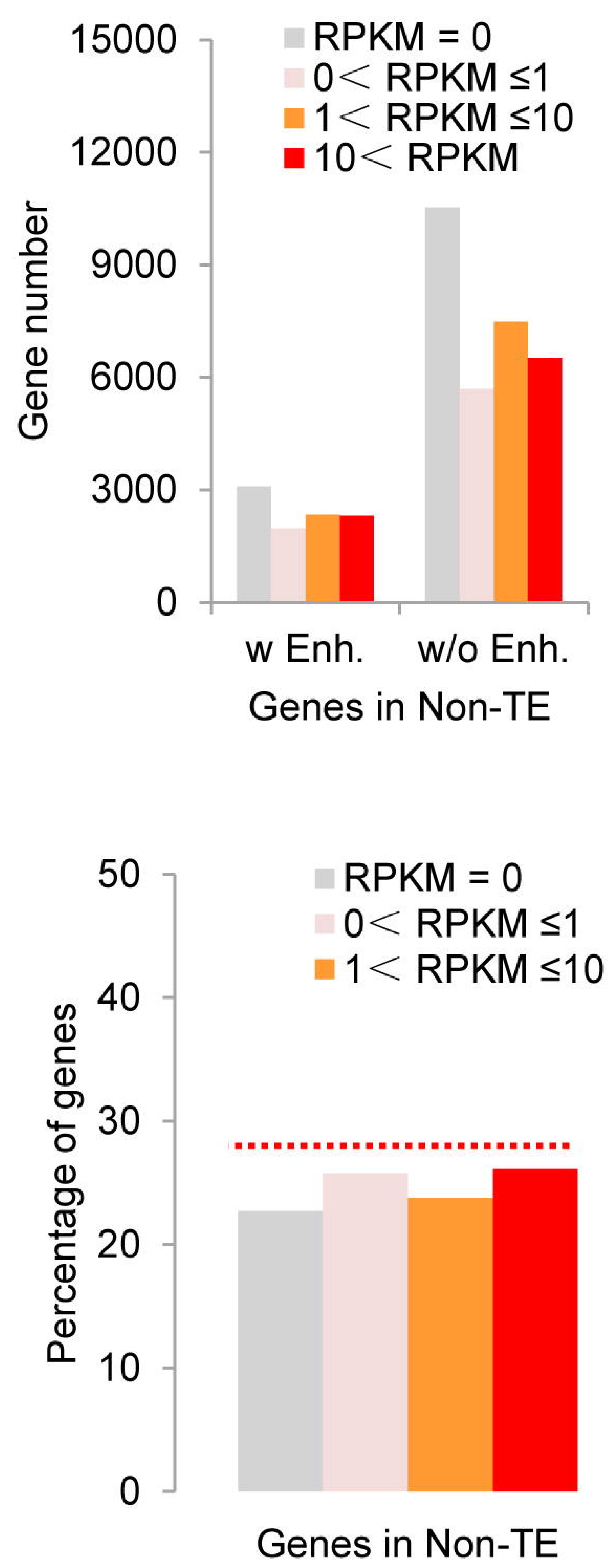
Figure 4, Sun et al.

A

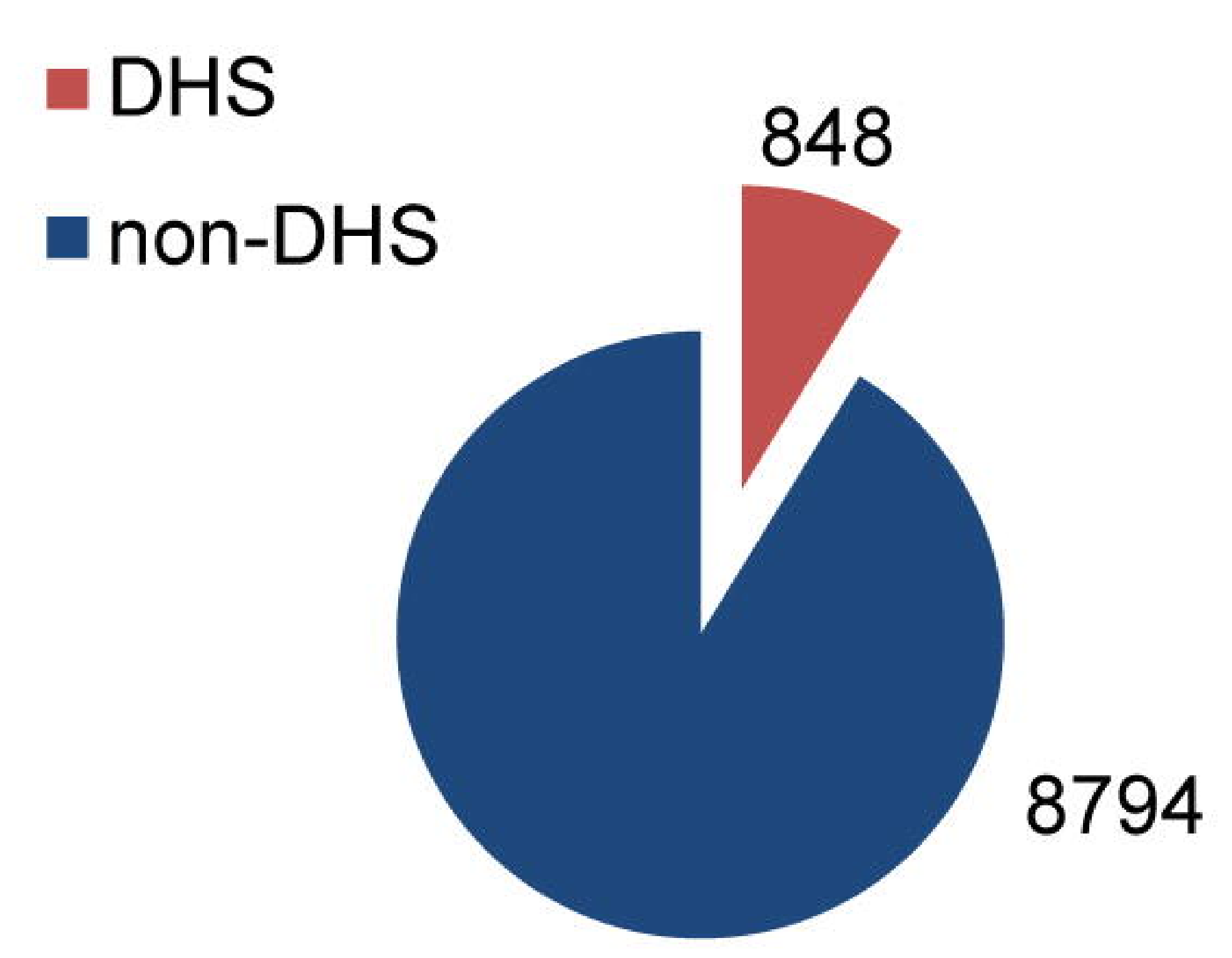

C

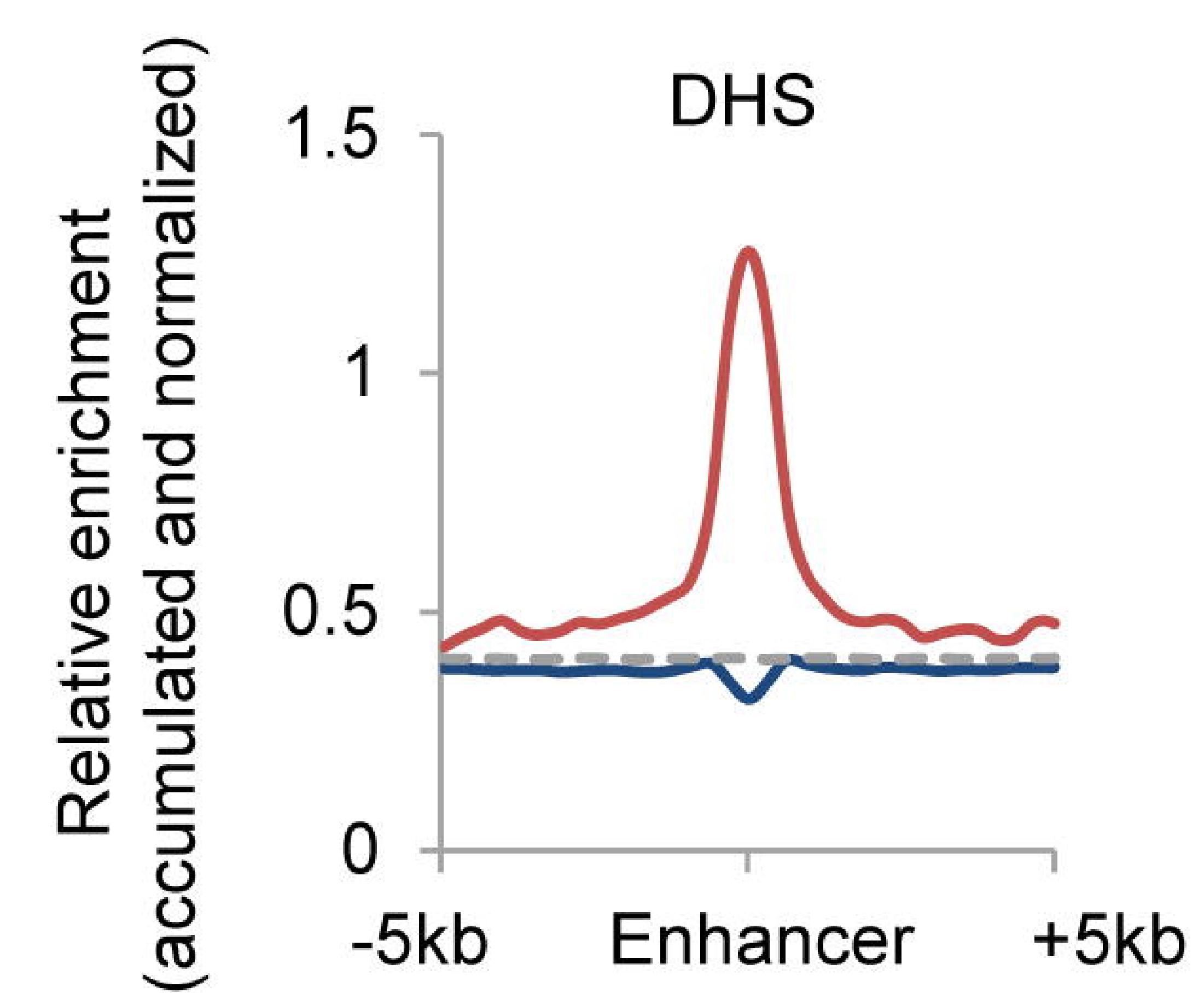

0.6

0.4

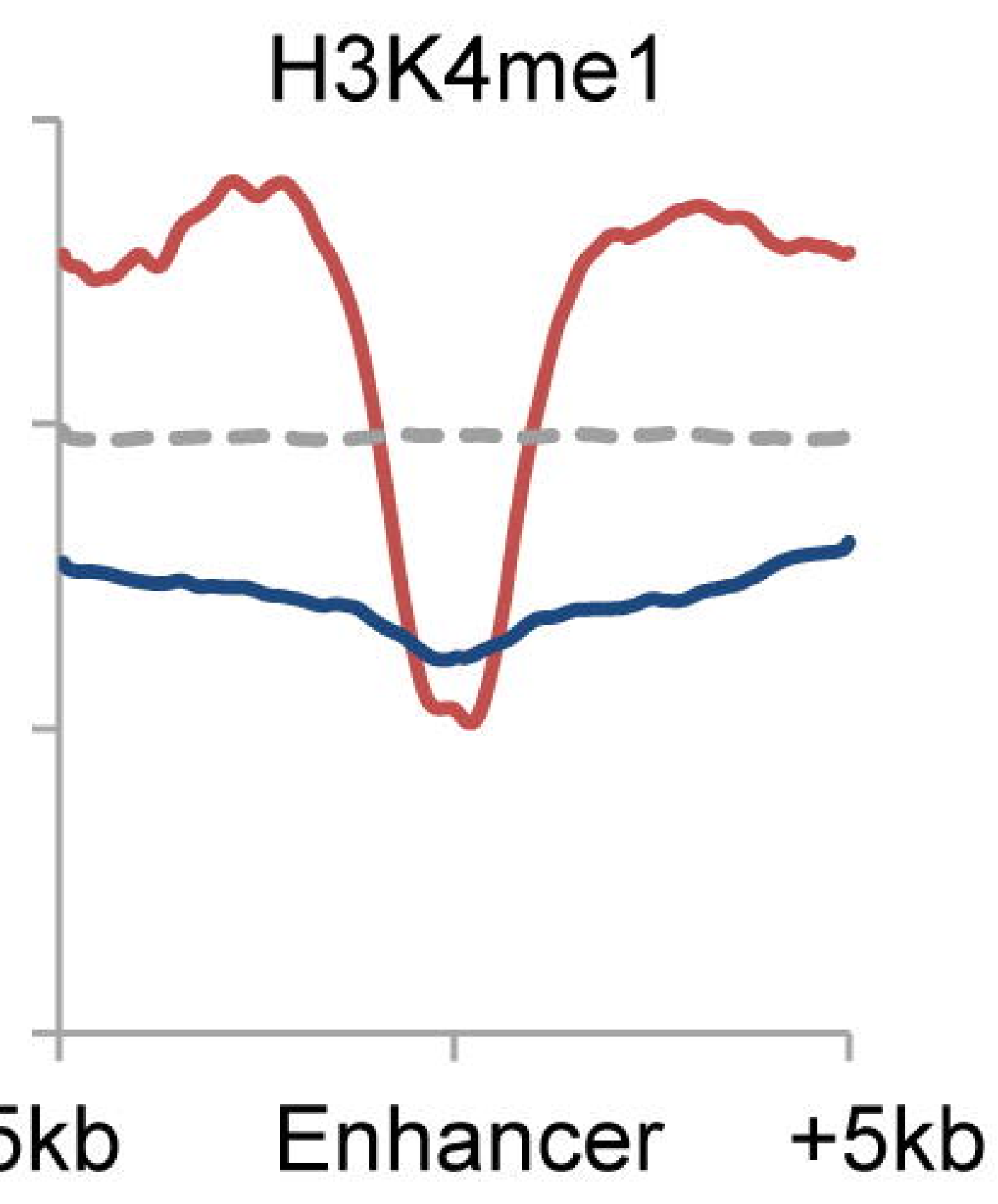

w DHSw non-DHS

Enh. Enh.

Replicate 2

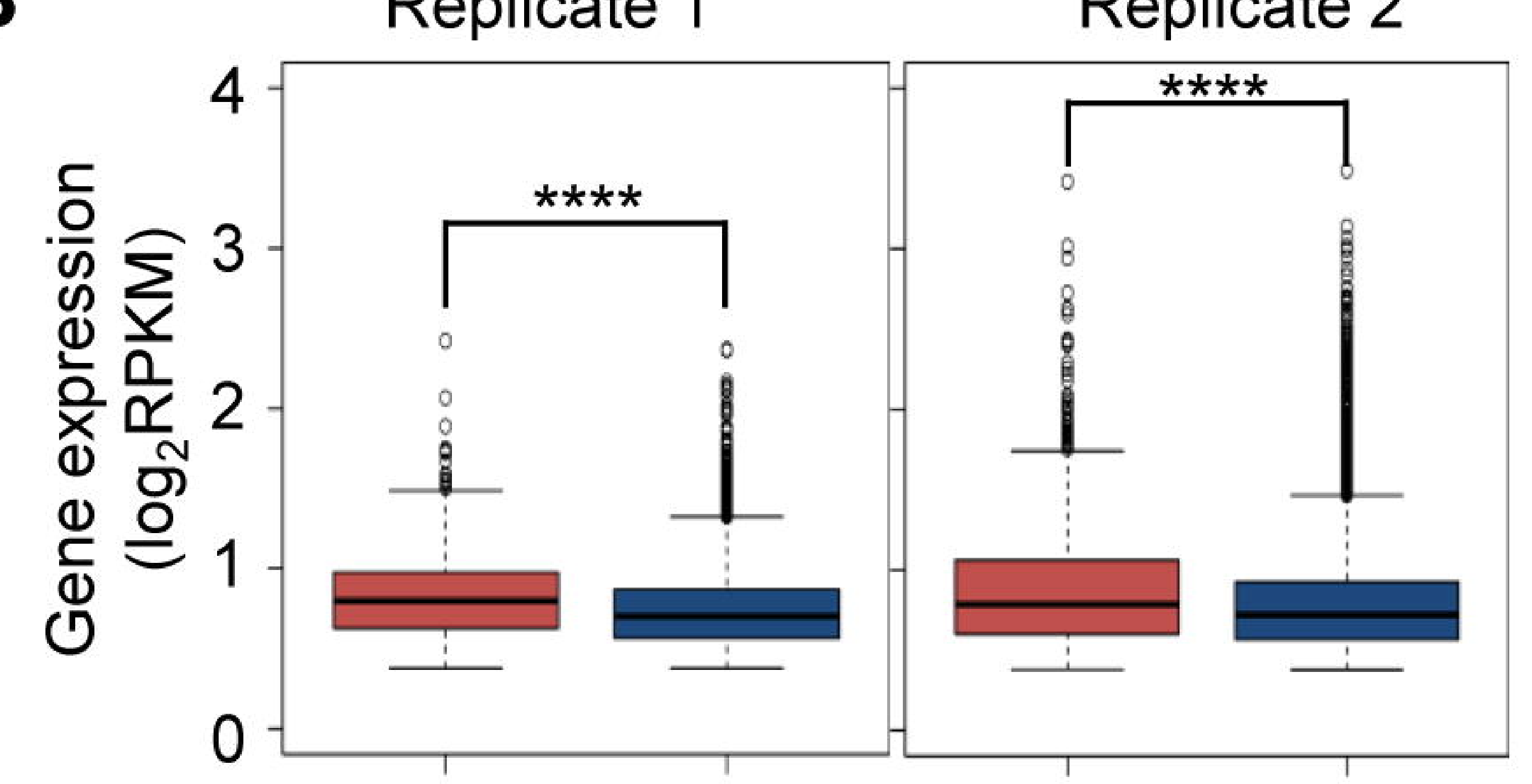

w DHSw non-DHS

Enh. Enh.
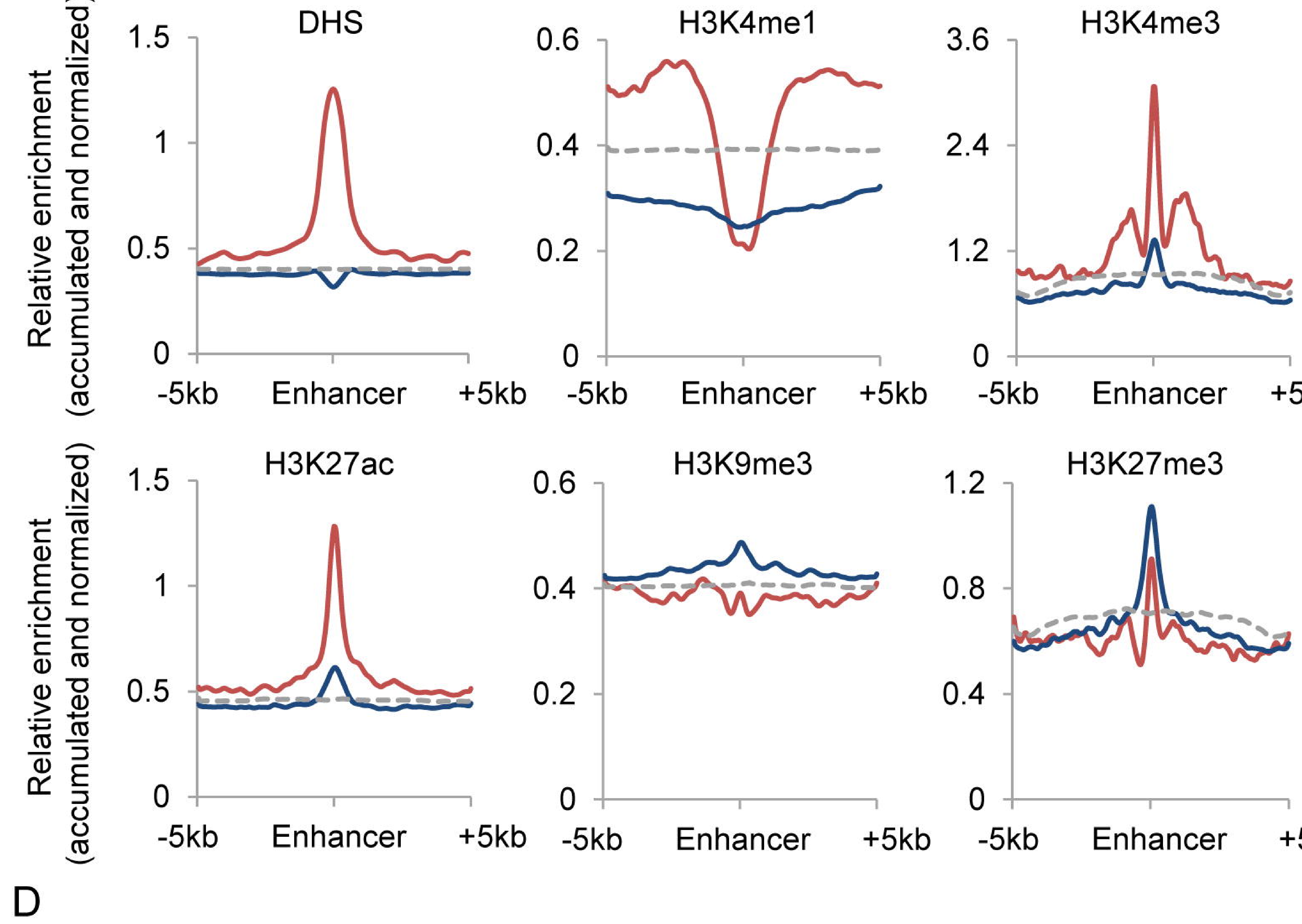

$-5 k b$ Enhancer $+5 k b$
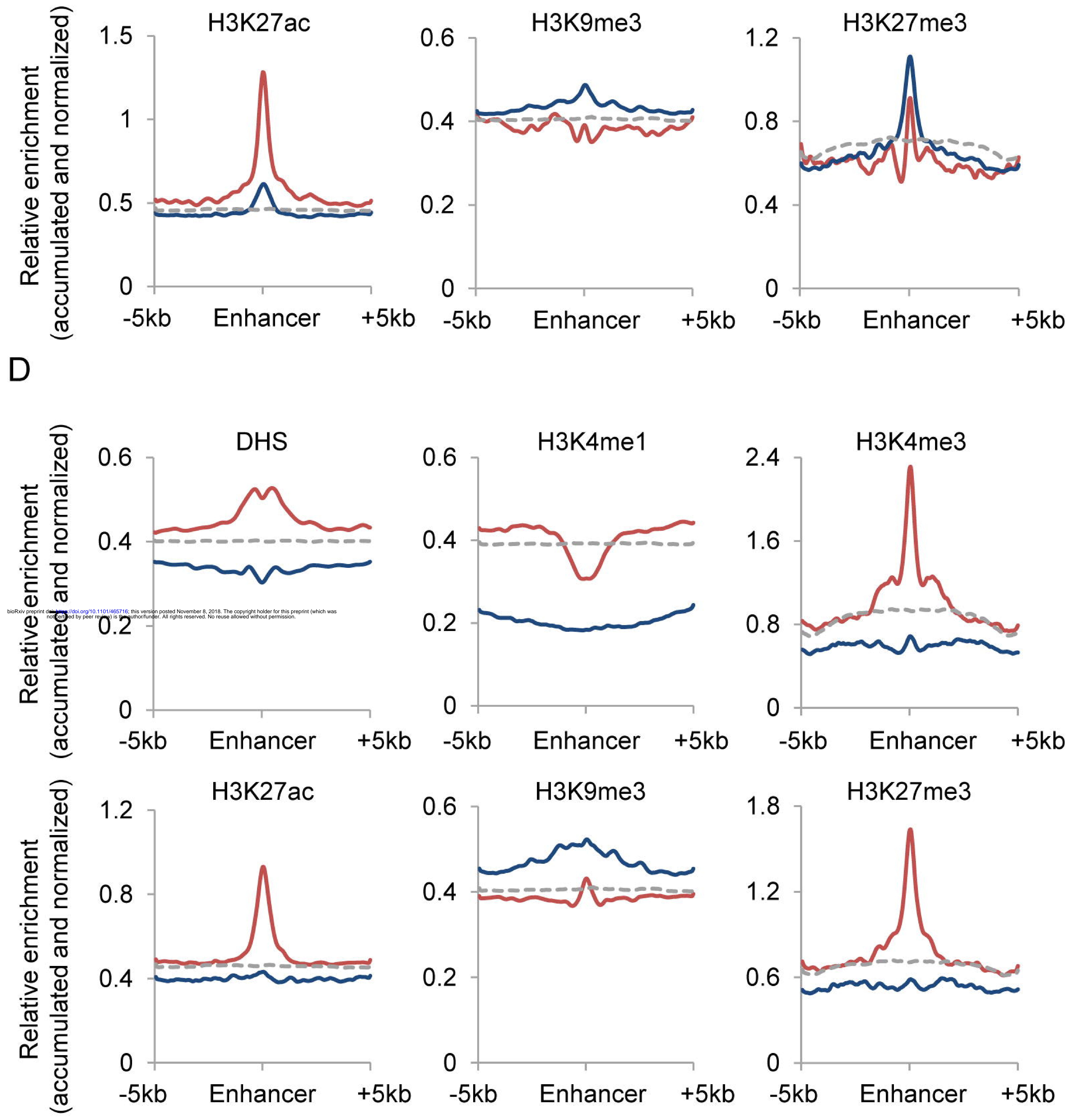
Figure 5, Sun et al.

A

$\begin{array}{lllllllll}\text { DHS } & \mathrm{C} 1 & \mathrm{sC} 2 & \mathrm{C} 3 & \mathrm{C} 4 & \mathrm{C} 5 & \mathrm{sC} 6 & \mathrm{C} 7 & \mathrm{C} 8\end{array}$

H3K4me1

H3K4me3

H3K27ac

H3K9me3

H3K27me3
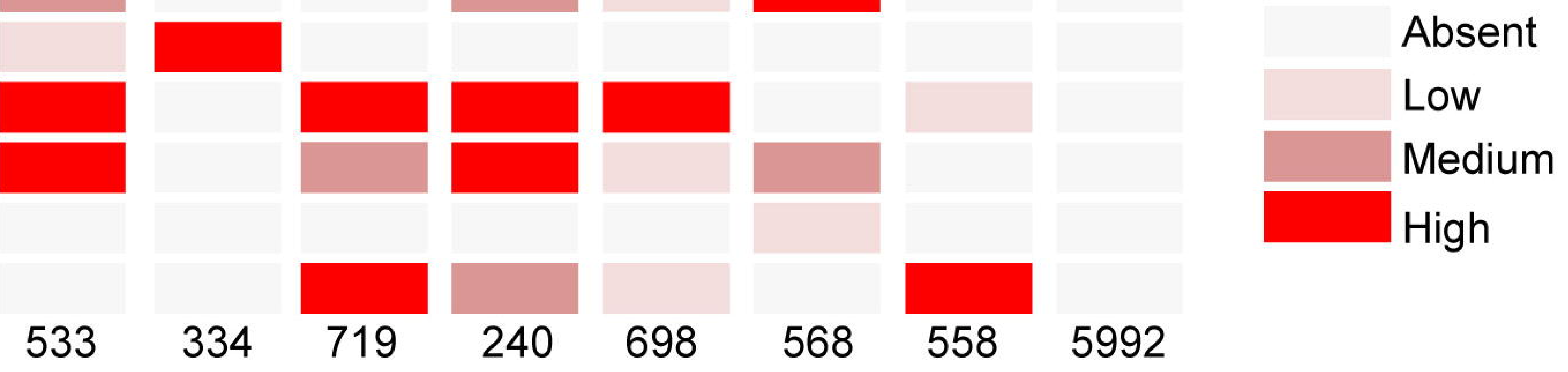

B

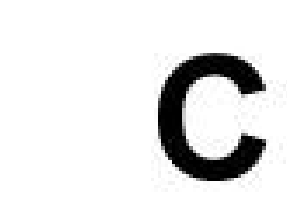

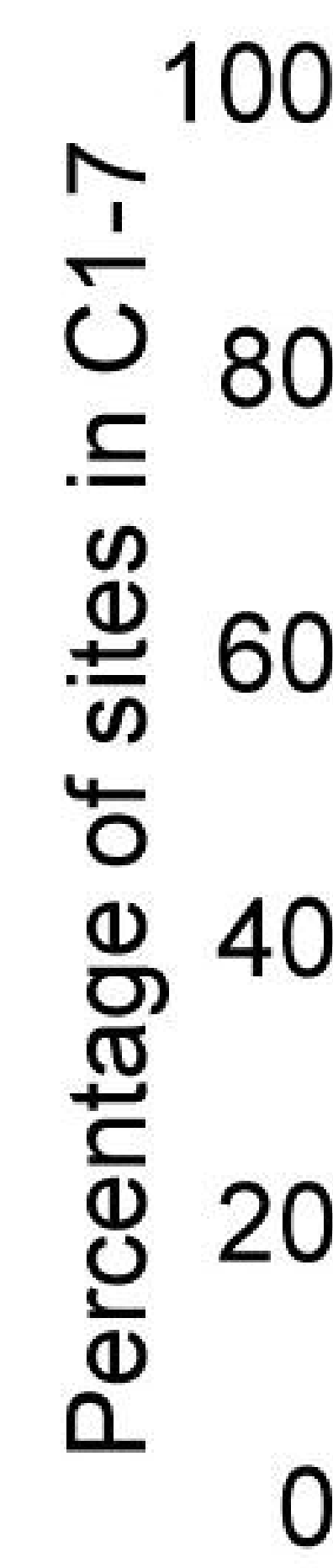

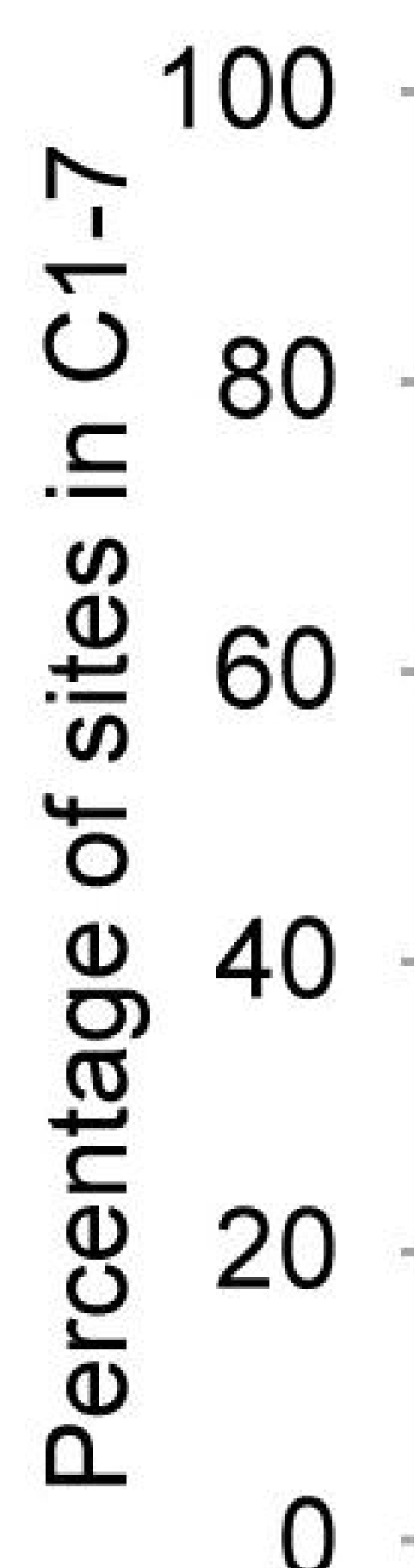

- High

Absent

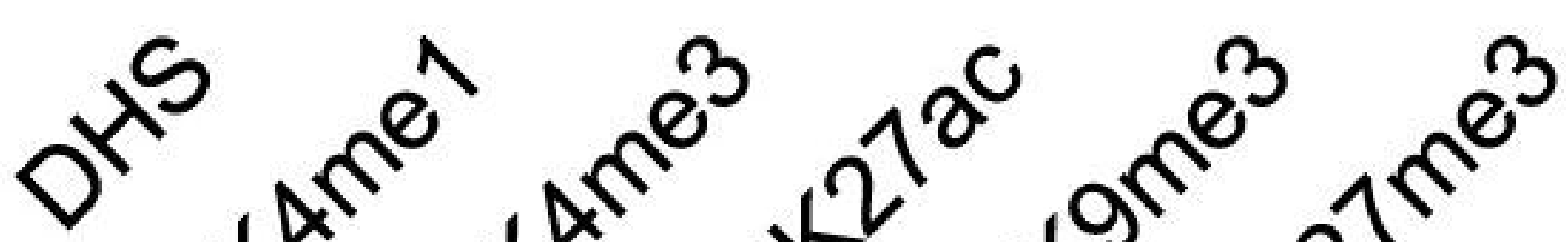

a

$\lambda^{3} t^{t^{2}}$
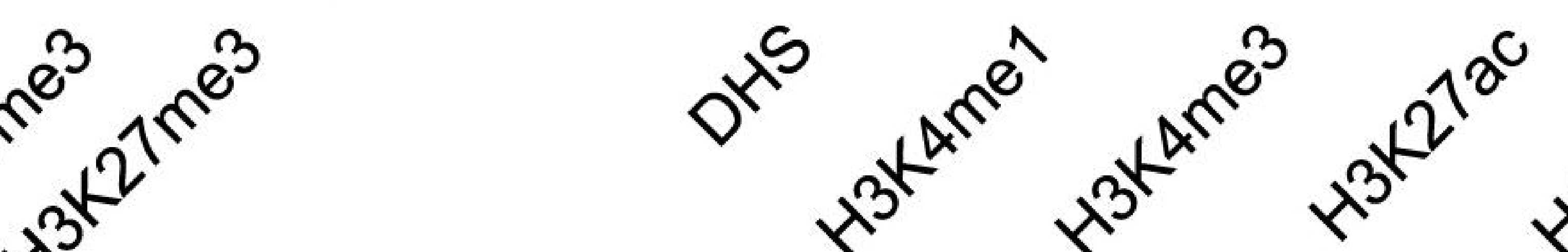

D

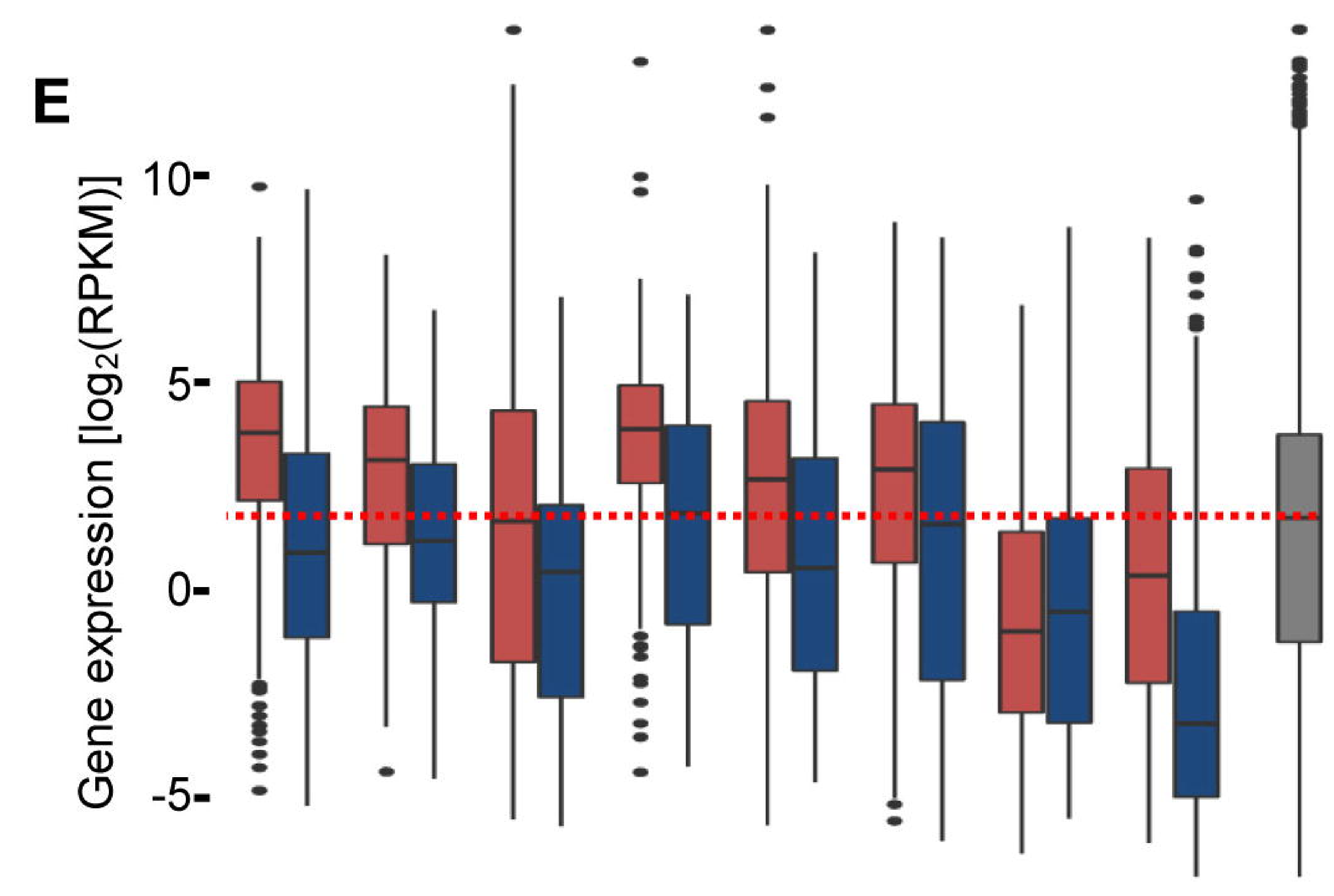

$\begin{array}{lllllllll}\mathrm{C} 1 & \mathrm{C} 2 & \mathrm{C} 3 & \mathrm{C} 4 & \mathrm{C} 5 & \mathrm{C} 6 & \mathrm{C} 7 & \mathrm{C} 8 & \text { Total }\end{array}$

0

C1-7

C8

Genes out of TE Genes in TE Total Genes 
A

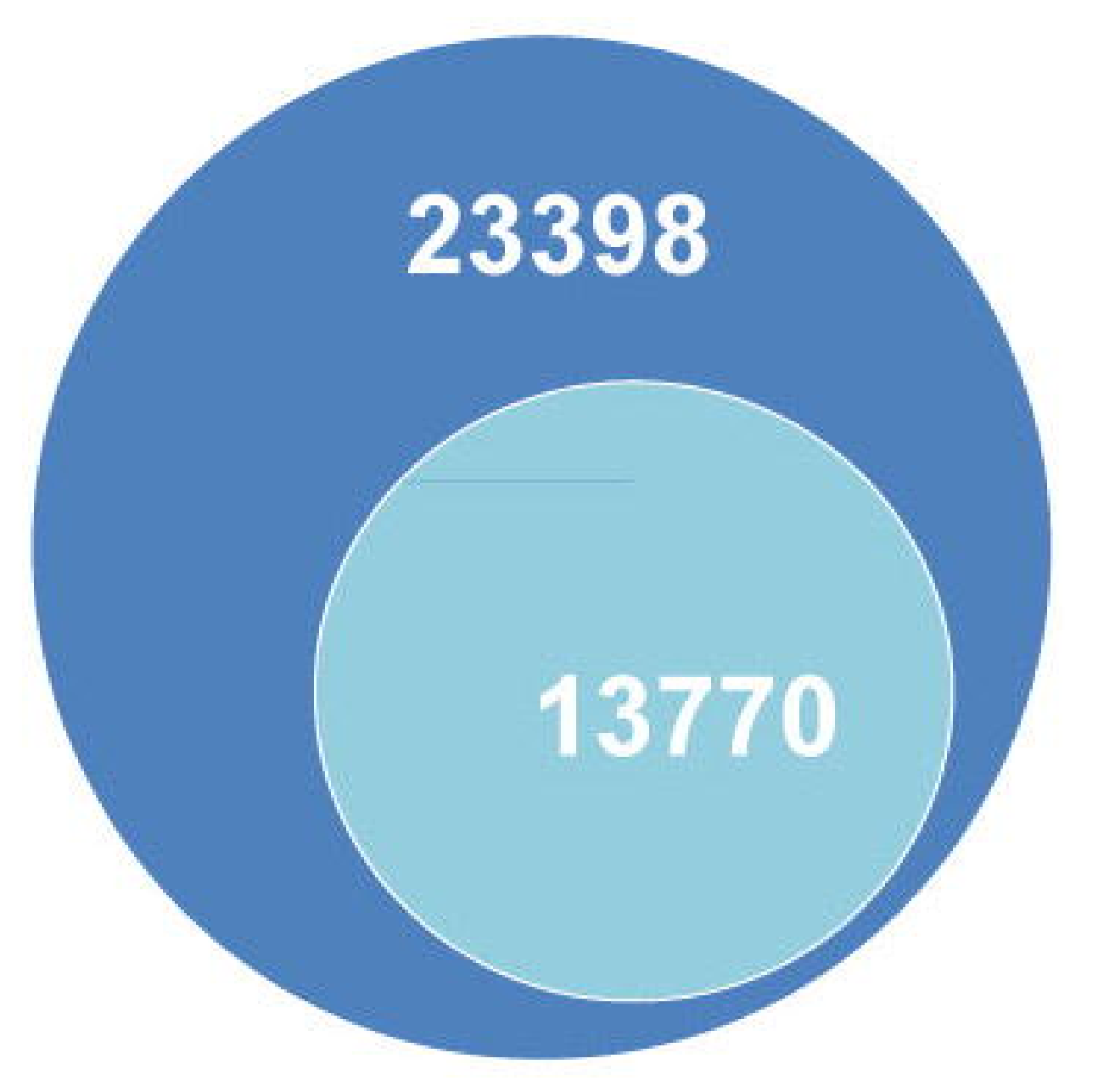

D

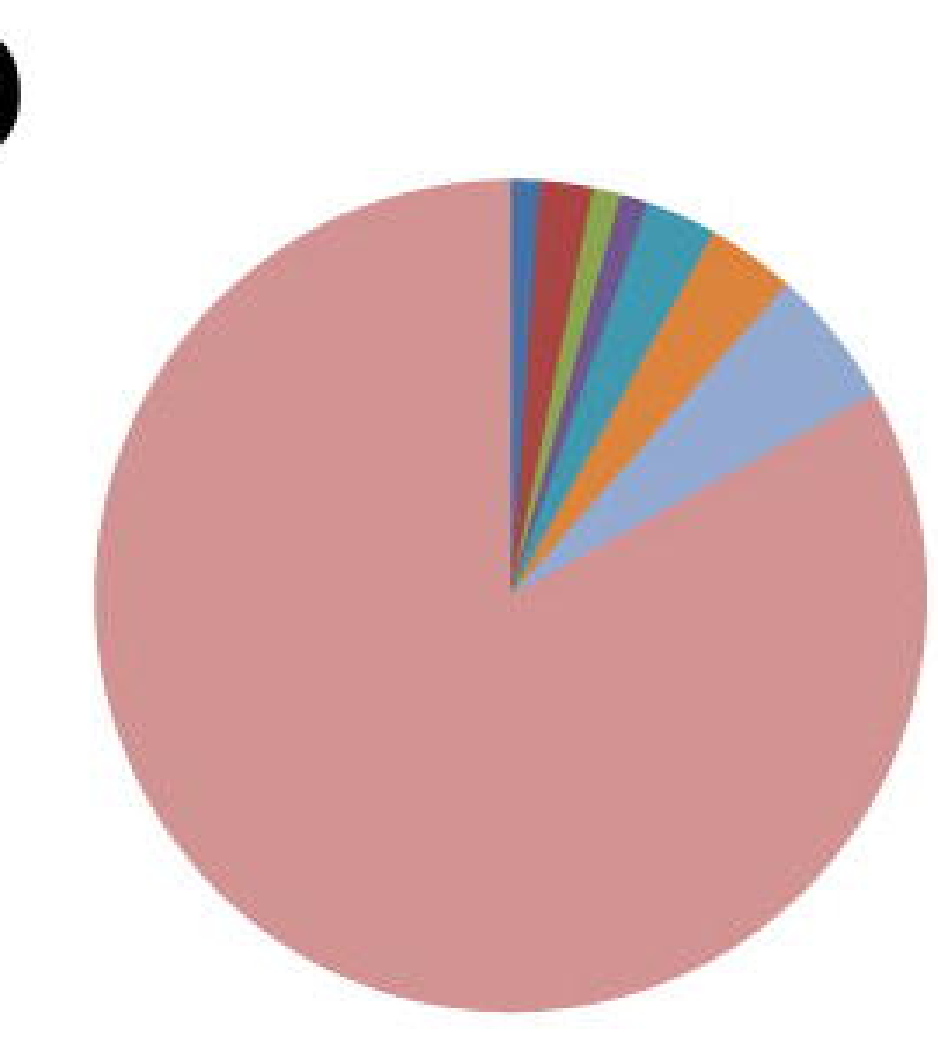

DHS enhancer

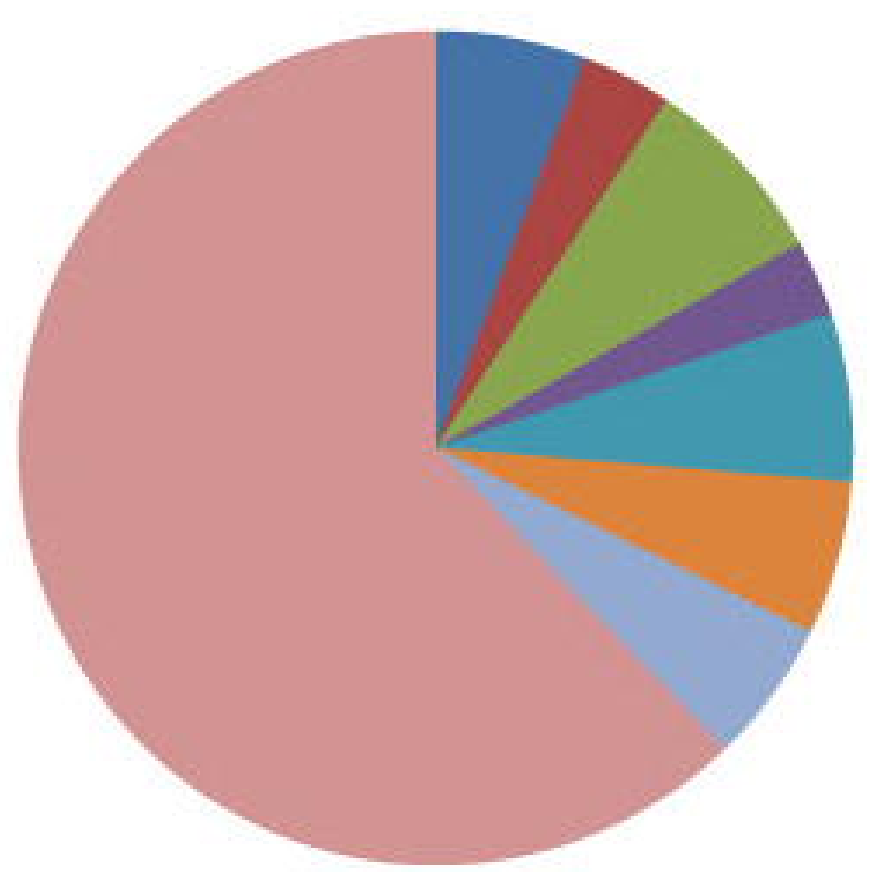

STARR-seq enhancer
B

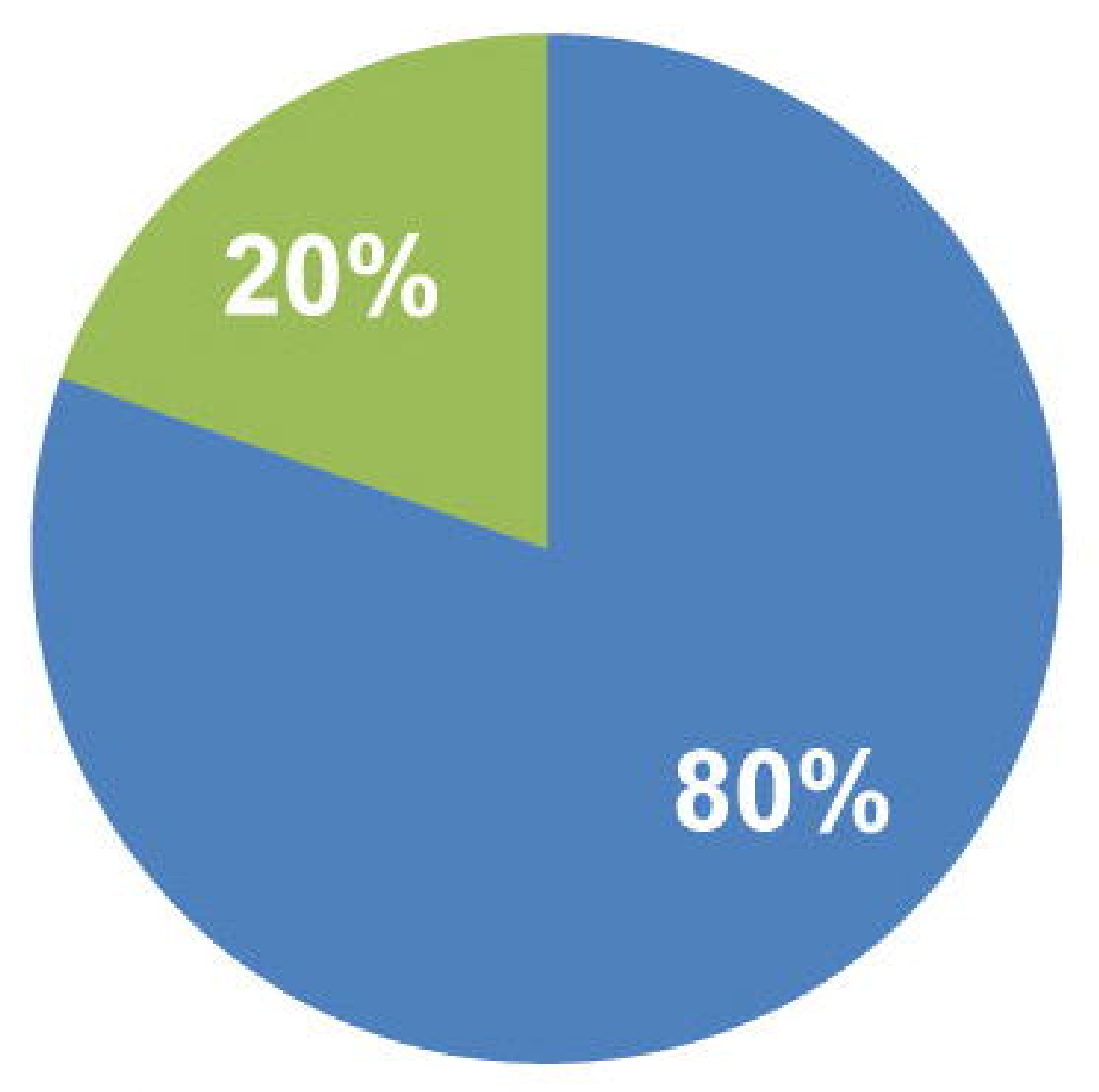

- intergenic $\square$ repeats
C

400

(418)

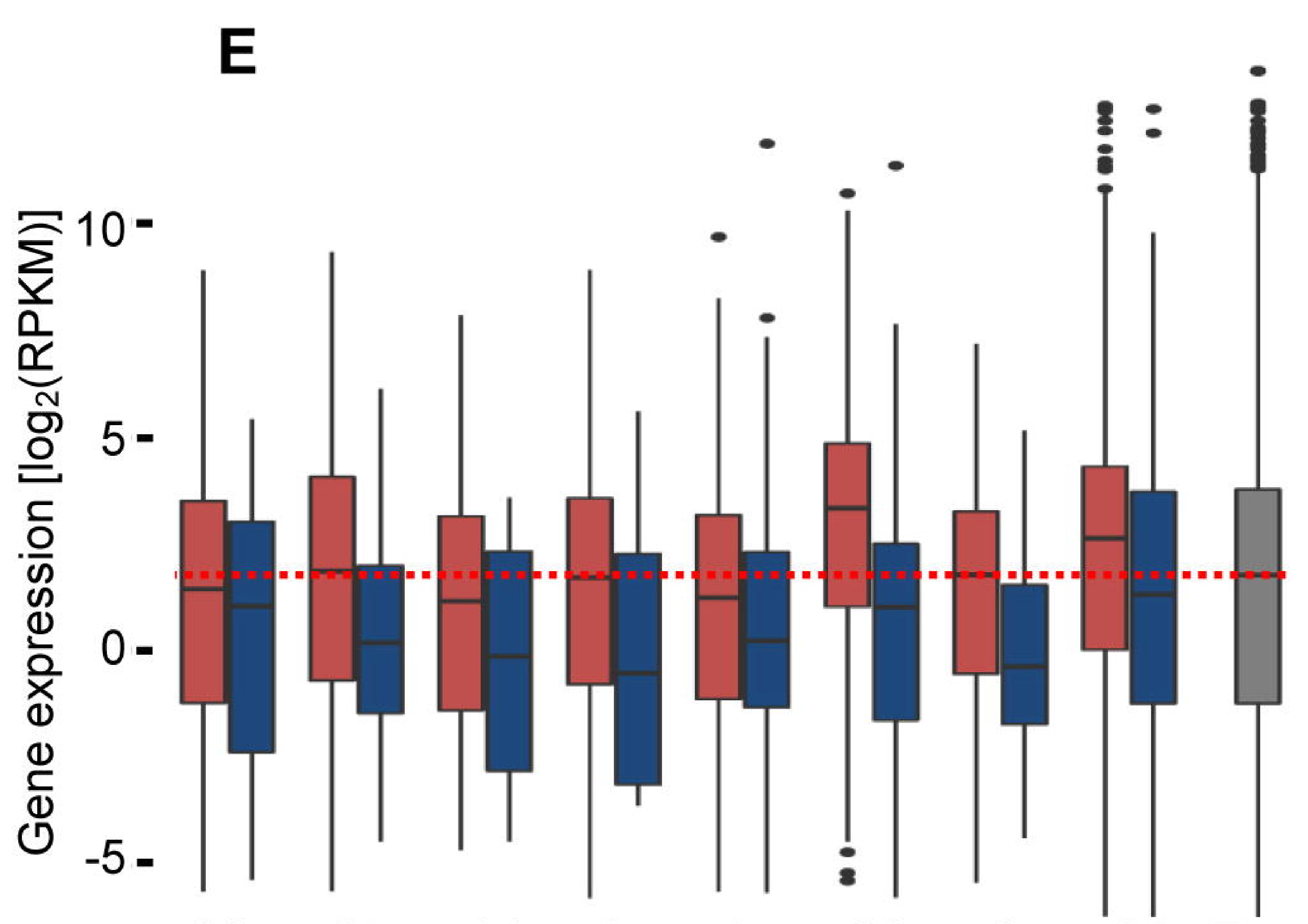

9242

C1

C2

C3

C4 C5 C6

C7

C1
C2
C3
C4
C5
C6
C7

$\mathbf{F}$

Genes out of TE Genes in TE Total Genes

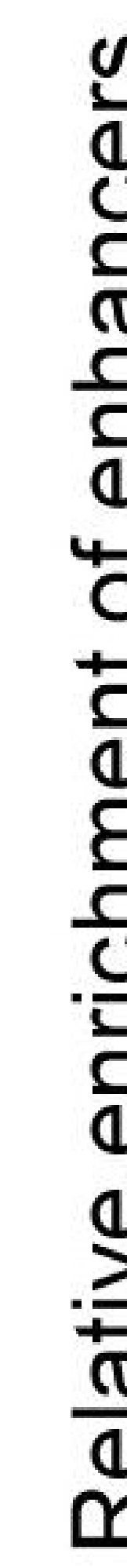

0.00
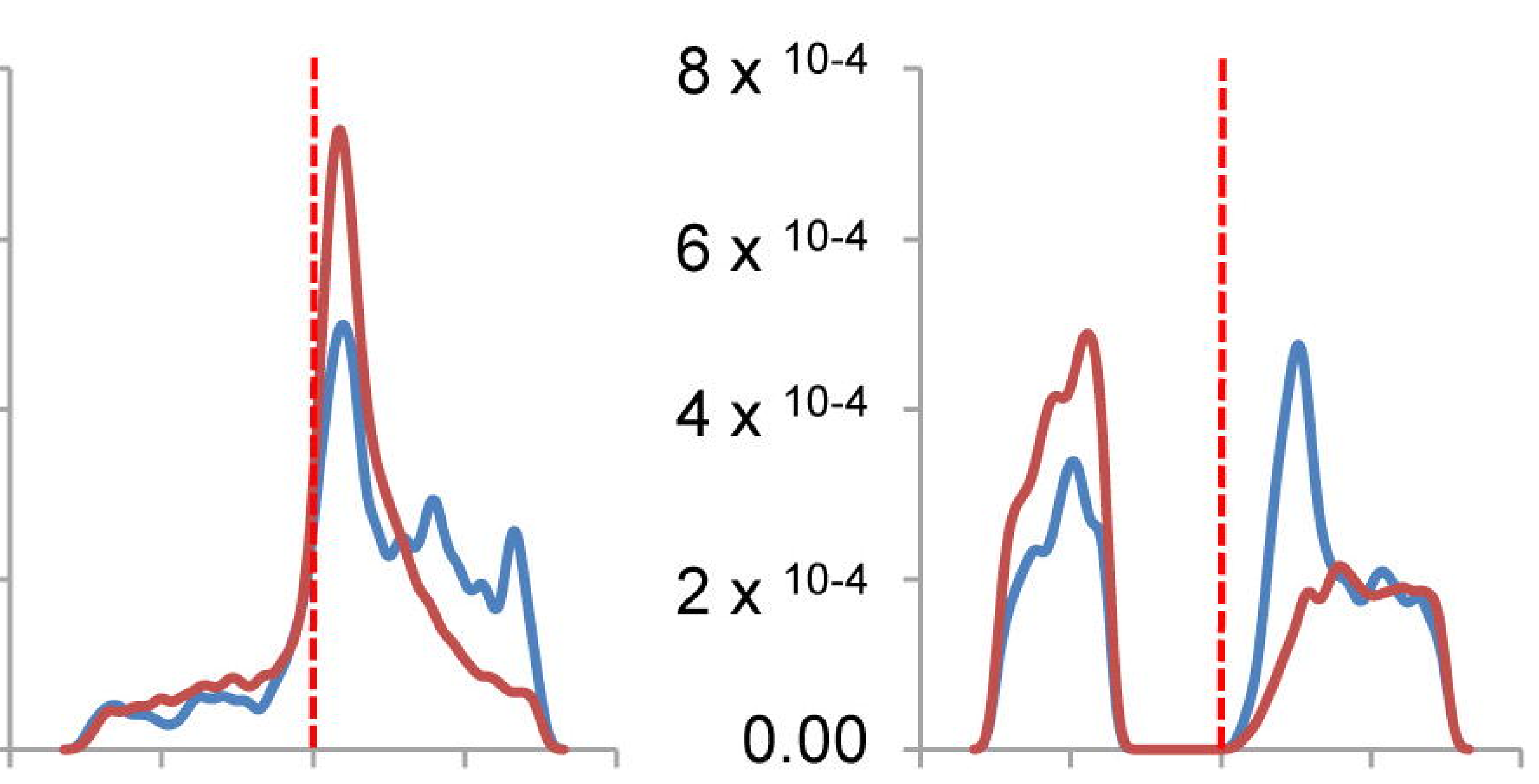

$2 \times^{10-3}$
$1 \times^{10-3}$
$5 \times^{10-3}$
0.00

$-4 k b-2 k b$ TSS $+2 k b+4 k b$

$-4 k b-2 k b$ TSS $+2 k b+4 k b$

DHS intergenic enhancer 
